\title{
Microlocal Asymptotic Analysis in Algebras of Generalized Functions
}

\author{
Antoine Delcroix \\ Equipe Analyse Algébrique Non Linéaire - Laboratoire $A O C$ \\ Faculté des sciences - Université des Antilles et de la Guyane, \\ BP 250, 97157 Pointe à Pitre Cedex, Guadeloupe (France) \\ Antoine.Delcroix@univ-ag.fr \\ Jean-André Marti \\ Equipe Analyse Algébrique Non Linéaire - Laboratoire G TS I \\ Faculté des sciences - Université des Antilles et de la Guyane, \\ BP 250, 97157 Pointe à Pitre Cedex, Guadeloupe (France) \\ Jean-Andre.Marti@univ-ag.fr \\ Michael Oberguggenberger* \\ Institute of Basic Sciences in Engineering - Unit of Engineering Mathematics \\ Faculty of Civil Engineering - University of Innsbruck, \\ Technikerstraße 13 - 6020 Innsbruck, Austria \\ Michael.Oberguggenberger@uibk.ac.at
}

April 9, 2007

\begin{abstract}
We introduce a new type of local and microlocal asymptotic analysis in algebras of generalized functions, based on the presheaf properties of those algebras and on the properties of their elements with respect to a regularizing parameter. Contrary to the more classical frequential analysis based on the Fourier transform, we can describe a singular asymptotic spectrum which has good properties with respect to nonlinear operations. In this spirit we give several examples of propagation of singularities through nonlinear operators.
\end{abstract}

Keywords: microlocal analysis, generalized functions, nonlinear operators, presheaf, propagation of singularities, singular spectrum.

Mathematics Subject Classification (2000): 35A18, 35A27, 46E10, 46F30, 46T30

\section{Introduction}

Various nonlinear theories of generalized functions have been developed over the past twenty years, with contributions by many authors. These theories have in common that the space of distributions is enlarged or embedded into algebras so that nonlinear operations on distributions become possible. These methods have been especially efficient in formulating and solving nonlinear differential problems with irregular data.

Most of the algebras of generalized functions possess the structure of sheaves or presheaves, which may contain some sub(pre)sheaves with particular properties. For example, the sheaf

${ }^{*}$ Supported by FWF (Austria), grant Y237. 
$\mathcal{G}$ of the special Colombeau algebras [2, 7, 15] contains the subsheaf $\mathcal{G}^{\infty}$ of so-called regular sections of $\mathcal{G}$ such that the embedding: $\mathcal{G}^{\infty} \rightarrow \mathcal{G}$ is the natural extension of the classical one: $\mathrm{C}^{\infty} \rightarrow \mathcal{D}^{\prime}$. This notion of regularity leads to $\mathcal{G}^{\infty}$-local or microlocal analysis of generalized functions, extending the classical results on the $\mathrm{C}^{\infty}$-microlocal analysis of distributions due to Hörmander [8]. This concept has been slightly extended in [4] to less restrictive kinds of measuring regularity. In [14], microlocal regularity theory in analytic and Gevrey classes has been generalized to algebras of generalized functions. Many results on propagation of singularities and pseudodifferential techniques have been obtained during the last years (see [5, 6, 9, 10, 11]). Nevertheless, these results are still mainly limited to linear cases, since they use frequential methods based on the Fourier transform.

In this paper, we develop a new type of asymptotic local and microlocal analysis of generalized functions in the framework of $(\mathcal{C}, \mathcal{E}, \mathcal{P})$-algebras [12, 13], following first steps undertaken in [12]. An example of the construction is given by taking $\mathcal{G}$ as a special case of a $(\mathcal{C}, \mathcal{E}, \mathcal{P})$ structure (see Subsection 2.2 for details). Let $\mathcal{F}$ be a subsheaf of vector spaces (or algebras) of $\mathcal{G}$ and $\left(u_{\varepsilon}\right)_{\varepsilon}$ a representative of $u \in \mathcal{G}(\Omega)$ for some open set $\Omega \subset \mathbb{R}^{n}$. We first define $\mathcal{O}_{\mathcal{G}}^{\mathcal{F}}(u)$ as the set of all $x \in \Omega$ such that $u_{\varepsilon}$ tends to a section of $\mathcal{F}$ above some neighborhood of $x$. The $\mathcal{F}$-singular support of $u$ is $\Omega \backslash \mathcal{O}_{\mathcal{G}}^{\mathcal{F}}(u)$. For fixed $x$ and $u, N_{x}(u)$ is the set of all $r \in \mathbb{R}_{+}$such that $\varepsilon^{r} u_{\varepsilon}$ tends to a section of $\mathcal{F}$ above some neighborhood of $x$. The $\mathcal{F}$-singular spectrum of $u$ is the set of all $(x, r) \in \Omega \times \mathbb{R}_{+}$such that $r \in \mathbb{R}_{+} \backslash N_{x}(u)$. It gives a spectral decomposition of the $\mathcal{F}$-singular support of $u$.

This asymptotic analysis is extended to $(\mathcal{C}, \mathcal{E}, \mathcal{P})$-algebras. This gives the general asymptotic framework, in which the net $\left(\varepsilon^{r}\right)_{\varepsilon}$ is replaced by any net $a$ satisfying some technical conditions, leading to the concept of the $(a, \mathcal{F})$-singular asymptotic spectrum. The main advantage is that this asymptotic analysis is compatible with the algebraic structure of the $(\mathcal{C}, \mathcal{E}, \mathcal{P})$-algebras. Thus, the $(a, \mathcal{F})$-singular asymptotic spectrum inherits good properties with respect to nonlinear operations (Theorem 15 and Corollary 16).

The paper is organized as follows. In Section 2, we introduce the sheaves of $(\mathcal{C}, \mathcal{E}, \mathcal{P})$-algebras and develop the local asymptotic analysis. Section 0 is devoted to the $(a, \mathcal{F})$-microlocal analysis and specially to the nonlinear properties of the $(a, \mathcal{F})$-singular asymptotic spectrum. In Section Q 1 various examples of the propagation of singularities through non linear differential operators are given.

\section{Preliminary definitions and local parametric analysis}

\subsection{The presheaves of $(\mathcal{C}, \mathcal{E}, \mathcal{P})$-algebras: the algebraic structure}

We begin by recalling the notions from [12, 13] that form the basis for our study.

(a) Let:

(1) $\Lambda$ be a set of indices;

(2) $A$ be a solid subring of the $\operatorname{ring} \mathbb{K}^{\Lambda}(\mathbb{K}=\mathbb{R}$ or $\mathbb{C})$; this means that whenever $\left(\left|s_{\lambda}\right|\right)_{\lambda} \leq\left(\left|r_{\lambda}\right|\right)_{\lambda}$ for some $\left(\left(s_{\lambda}\right)_{\lambda},\left(r_{\lambda}\right)_{\lambda}\right) \in \mathbb{K}^{\Lambda} \times A$, that is, $\left|s_{\lambda}\right| \leq\left|r_{\lambda}\right|$ for all $\lambda$, it follows that $\left(s_{\lambda}\right)_{\lambda} \in A$;

(3) $I_{A}$ be a solid ideal of $A$;

(4) $\mathcal{E}$ be a sheaf of $\mathbb{K}$-topological algebras over a topological space $X$.

Moreover, suppose that

(5) for any open set $\Omega$ in $X$, the algebra $\mathcal{E}(\Omega)$ is endowed with a family $\mathcal{P}(\Omega)=\left(p_{i}\right)_{i \in I(\Omega)}$ of semi-norms such that if $\Omega_{1}, \Omega_{2}$ are two open subsets of $X$ with $\Omega_{1} \subset \Omega_{2}$, it follows that $I\left(\Omega_{1}\right) \subset I\left(\Omega_{2}\right)$ and if $\rho_{1}^{2}$ is the restriction operator $\mathcal{E}\left(\Omega_{2}\right) \rightarrow \mathcal{E}\left(\Omega_{1}\right)$, then, for each $p_{i} \in \mathcal{P}\left(\Omega_{1}\right)$ the semi-norm $\widetilde{p}_{i}=p_{i} \circ \rho_{1}^{2}$ extends $p_{i}$ to $\mathcal{P}\left(\Omega_{2}\right)$.

(6) Let $\Theta=\left(\Omega_{h}\right)_{h \in H}$ be any family of open sets in $X$ with $\Omega=\cup_{h \in H} \Omega_{h}$. Then, for each $p_{i} \in \mathcal{P}(\Omega), i \in I(\Omega)$, there exist a finite subfamily of $\Theta: \Omega_{1}, \ldots, \Omega_{n(i)}$ and corresponding 
semi-norms $p_{1} \in \mathcal{P}\left(\Omega_{1}\right), \ldots, p_{n(i)} \in \mathcal{P}\left(\Omega_{n(i)}\right)$, such that, for any $u \in \mathcal{E}(\Omega)$

$$
p_{i}(u) \leq p_{1}\left(\left.u\right|_{\Omega_{1}}\right)+\ldots+p_{n(i)}\left(\left.u\right|_{\Omega_{n(i)}}\right) .
$$

(b) Define $|B|=\left\{\left(\left|r_{\lambda}\right|\right)_{\lambda},\left(r_{\lambda}\right)_{\lambda} \in B\right\}, B=A$ or $I_{A}$, and set

$$
\begin{gathered}
\mathcal{H}_{(A, \mathcal{E}, \mathcal{P})}(\Omega)=\left\{\left(u_{\lambda}\right)_{\lambda} \in[\mathcal{E}(\Omega)]^{\Lambda} \mid \forall i \in I(\Omega),\left(\left(p_{i}\left(u_{\lambda}\right)\right)_{\lambda} \in|A|\right\}\right. \\
\mathcal{J}_{\left(I_{A}, \mathcal{E}, \mathcal{P}\right)}(\Omega)=\left\{\left(u_{\lambda}\right)_{\lambda} \in[\mathcal{E}(\Omega)]^{\Lambda}\left|\forall i \in I(\Omega),\left(p_{i}\left(u_{\lambda}\right)\right)_{\lambda} \in\right| I_{A} \mid\right\} \\
\mathcal{C}=A / I_{A},
\end{gathered}
$$

Note that, from (2), $|A|$ is a subset of $A$ and that $A_{+}=\left\{\left(b_{\lambda}\right)_{\lambda} \in A, \forall \lambda \in \Lambda, b_{\lambda} \geq 0\right\}=|A|$. The same holds for $I_{A}$. Furthermore, (2) implies also that $A$ is a $\mathbb{K}$-algebra. Indeed, it suffices to show that $A$ is stable under multiplication by elements of $\mathbb{K}$. Let $c$ be in $\mathbb{K}$ and $\left(a_{\lambda}\right)_{\lambda} \in A$. Then $\left(c a_{\lambda}\right)_{\lambda}$ satisfies $\left(\left|c a_{\lambda}\right|\right)_{\lambda} \leq\left(\left|n a_{\lambda}\right|\right)_{\lambda}$ for some $n \in \mathbb{N}$. We have $\left(n a_{\lambda}\right)_{\lambda} \in A$ since $A$ is stable under addition. Thus, using $(2)$, we get that $\left(c a_{\lambda}\right)_{\lambda} \in A$.

For later reference, we recall the following notions entering in the definition of a sheaf $\mathcal{A}$ on $X$. Let $\left(\Omega_{h}\right)_{h \in H}$ be a family of open sets in $X$ with $\Omega=\cup_{h \in H} \Omega_{h}$.

$\left(F_{1}\right)$ (Localization principle) Let $u, v \in \mathcal{A}(\Omega)$. If all restrictions $\left.u\right|_{\Omega_{h}}$ and $\left.u\right|_{\Omega_{h}}, h \in H$, coincide, then $u=v$ in $\mathcal{A}(\Omega)$.

$\left(F_{2}\right)$ (Gluing principle) Let $\left(u_{h}\right)_{h \in H}$ be a coherent family of elements of $\mathcal{A}\left(\Omega_{h}\right)$, that is, the restrictions to the non-void intersections of the $\Omega_{h}$ coincide. Then there is an element $u \in \mathcal{A}(\Omega)$ such that $\left.u\right|_{\Omega_{h}}=u_{h}$ for all $h \in H$.

Proposition 1 (i) $\mathcal{H}_{(A, \mathcal{E}, \mathcal{P})}$ is a sheaf of $\mathbb{K}$-subalgebras of the sheaf $\mathcal{E}^{\Lambda}$; (ii) $\mathcal{J}_{\left(I_{A}, \mathcal{E}, \mathcal{P}\right)}$ is a sheaf of ideals of $\mathcal{H}_{(A, \mathcal{E}, \mathcal{P})}$.

Proof. The proof can be found in [12, 13], so we just recall the main steps. We start from the statement that $\mathcal{E}$ and $\mathcal{E}^{\Lambda}$ are already sheaves of algebras. From (5), we infer that $\mathcal{H}_{(A, \mathcal{E}, \mathcal{P})}$ and $\mathcal{J}_{\left(I_{A}, \mathcal{E}, \mathcal{P}\right)}$ are a presheaves (the restriction property holds) and that the localization property $\left(F_{1}\right)$ is valid. To obtain the gluing property $\left(F_{2}\right)$ we need property $(6)$, which generalizes the situation from $C^{\infty}$ to $\mathcal{E}$.

Theorem 2 The factor $\mathcal{H}_{(A, \mathcal{E}, \mathcal{P})} / \mathcal{J}_{\left(I_{A}, \mathcal{E}, \mathcal{P}\right)}$ is a presheaf satisfying the localization principle $\left(F_{1}\right)$.

Proof. From the previous proposition, we know that $\mathcal{A}=\mathcal{H}_{(A, \mathcal{E}, \mathcal{P})} / \mathcal{J}_{\left(I_{A}, \mathcal{E}, \mathcal{P}\right)}$ is a presheaf. For $\Omega_{1} \subset \Omega_{2}$, the restriction is defined by

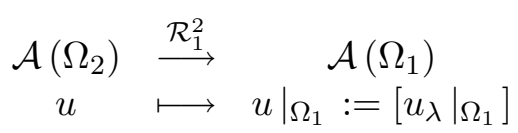

where $\left(u_{\lambda}\right)_{\lambda}$ is any representative of $u \in \mathcal{A}\left(\Omega_{2}\right)$ and $\left[u_{\lambda} \mid \Omega_{1}\right]$ denotes the class of $\left(u_{\lambda} \mid \Omega_{1}\right)_{\lambda}$. The definition is consistent and independent of the representative because for each $\left(u_{\lambda}\right)_{\lambda \in \Lambda} \in$ $\mathcal{H}_{(A, \mathcal{E}, \mathcal{P})}\left(\Omega_{2}\right)$ and $\left(\eta_{\lambda}\right)_{\lambda \in \Lambda} \in \mathcal{J}_{\left(I_{A}, \mathcal{E}, \mathcal{P}\right)}\left(\Omega_{2}\right)$, we have

$$
\left.\left(u_{\lambda}\right)_{\lambda}\right|_{\Omega_{1}}:=\left(\left.u_{\lambda}\right|_{\Omega_{1}}\right)_{\lambda} \in \mathcal{H}_{(A, \mathcal{E}, \mathcal{P})}\left(\Omega_{1}\right),\left.\quad\left(\eta_{\lambda}\right)_{\lambda}\right|_{\Omega_{1}}:=\left(\left.\eta_{\lambda}\right|_{\Omega_{1}}\right)_{\lambda} \in \mathcal{J}_{\left(I_{A}, \mathcal{E}, \mathcal{P}\right)}\left(\Omega_{1}\right)
$$

The localization principle is also obviously fulfilled because $\mathcal{J}_{\left(I_{A}, \mathcal{E}, \mathcal{P}\right)}$ is itself a sheaf.

Proposition 3 Under the hypothesis (2), the constant sheaf $\mathcal{H}_{(A, \mathbb{K},|\cdot|)} / \mathcal{J}_{\left(I_{A}, \mathbb{K},|\cdot|\right)}$ is exactly the $\operatorname{ring} \mathcal{C}=A / I_{A}$. 
Proof. We clearly have $\mathcal{H}_{(A, \mathbb{K},|\cdot|)}=A$ and $\mathcal{J}_{\left(I_{A}, \mathbb{K},|\cdot|\right)}=I_{A}$.

Definition 1 The factor presheaf of algebras over the ring $\mathcal{C}=A / I_{A}$ :

$$
\mathcal{A}=\mathcal{H}_{(A, \mathcal{E}, \mathcal{P})} / \mathcal{J}_{\left(I_{A}, \mathcal{E}, \mathcal{P}\right)}
$$

is called a presheaf of $(\mathcal{C}, \mathcal{E}, \mathcal{P})$-algebras.

Notation 1 We denote by $\left[u_{\lambda}\right]$ the class in $\mathcal{A}(\Omega)$ defined by $\left(u_{\lambda}\right)_{\lambda \in \Lambda} \in \mathcal{H}_{(A, \mathcal{E}, \mathcal{P})}(\Omega)$. For $u \in \mathcal{A}$, the notation $\left(u_{\lambda}\right)_{\lambda \in \Lambda} \in u$ means that $\left(u_{\lambda}\right)_{\lambda \in \Lambda}$ is a representative of $u$.

Remark 1 The problem of rendering $\mathcal{A}$ a sheaf (and even a fine sheaf) is not studied here. It is well known that the Colombeau algebra $\mathcal{G}$, which is a special case of a $(\mathcal{C}, \mathcal{E}, \mathcal{P})$-algebra (see Subsection 2.7), forms a fine sheaf [1, प]. The sheaf property can be inferred from the existence of a $C^{\infty}$-partition of unity associated to any open covering of an open set $\Omega$ of $\mathbb{R}^{d}$. This existence is fulfilled because $X=\mathbb{R}^{d}$ is a locally compact Hausdorff space. On the other hand, $C^{\infty}$ is a fine sheaf because multiplication by a smooth function defines a sheaf homomorphism in a natural way. Hence the usual topology and $C^{\infty}$-partition of unity defines the required sheaf partition of unity. Observing that $\mathcal{G}$ is a sheaf of $C^{\infty}$-modules and using the well known result that a sheaf of modules on a fine sheaf is itself a fine sheaf, we obtain the corresponding assertion about $\mathcal{G}$. In the general case, turning $\mathcal{A}$ into a sheaf requires additional hypotheses, which are not necessary for the results in this paper. Indeed, the presheaf structure of $\mathcal{A}$ and the $\left(F_{1}\right)$-principle are sufficient to develop our local and microlocal asymptotic analysis.

Remark 2 The map $\iota: \mathbb{K} \rightarrow A$ defined by $\iota(r)=(r)_{\lambda}$ is an embedding of algebras and induces a ring morphism from $\mathbb{K} \rightarrow \mathcal{C}$ if, and only if, $A$ is unitary (Lemma 14, 197). Indeed, if $A$ is unitary, $(r)_{\lambda}=r\left(1_{\lambda}\right)_{\lambda}$ is an element of $A$ since $A$ is a $\mathbb{K}$-algebra, and $\iota$ is clearly an injective ring morphism. The converse is obvious. Moreover, if $\Lambda$ is a directed set with partial order relation $\prec$ and if

$$
I_{A} \subset\left\{\left(a_{\lambda}\right)_{\lambda} \in A \mid \lim _{\Lambda} a_{\lambda}=0\right\}
$$

then the morphism $\iota$ is injective. Indeed, if $[\iota(r)]=0$, relation (1) implies that the limit of the constant sequence $(r)_{\lambda}$ is null, thus $r=0$.

\subsection{Relationship with distribution theory and Colombeau algebras}

One main feature of this construction is that we can choose the triple $(\mathcal{C}, \mathcal{E}, \mathcal{P})$ such that the sheaves $\mathrm{C}^{\infty}$ and $\mathcal{D}^{\prime}$ are embedded in the corresponding sheaf $\mathcal{A}$. In particular, we can multiply (the images of) distributions in $\mathcal{A}$.

We consider the sheaf $\mathcal{E}=\mathrm{C}^{\infty}$ over $\mathbb{R}^{d}$, where $\mathcal{P}$ is the usual family of topologies $\left(\mathcal{P}_{\Omega}\right)_{\Omega \in \mathcal{O}\left(\mathbb{R}^{d}\right)}$. Here $\mathcal{O}\left(\mathbb{R}^{d}\right)$ denotes the set of all open sets of $\mathbb{R}^{d}$; this notation will be used in the sequel. Let us recall that $\mathcal{P}_{\Omega}$ is defined by the family of semi-norms $\left(p_{K, l}\right)_{K \Subset \Omega, l \in \mathbb{N}}$ with

$$
\forall f \in \mathrm{C}^{\infty}(\Omega), \quad p_{K, l}(f)=\sup _{x \in K,|\alpha| \leq l}\left|\partial^{\alpha} f(x)\right| .
$$

From Lemma 14 in [13], it follows that the canonical maps, defined for any $\Omega \in \mathcal{O}\left(\mathbb{R}^{d}\right)$ by

$$
\sigma_{\Omega}: \mathrm{C}^{\infty}(\Omega) \rightarrow \mathcal{H}_{(A, \mathcal{E}, \mathcal{P})}(\Omega) \quad f \mapsto(f)_{\lambda},
$$

are injective morphism of algebras if, and only if, $A$ is unitary. Under this assumption, these maps give rise to a canonical sheaf embedding of $\mathrm{C}^{\infty}$ into $\mathcal{H}_{(A, \mathcal{E}, \mathcal{P})}$ and (using a partition of 
unity in $\mathrm{C}^{\infty}$ inducing a sheaf structure on $\mathcal{A}$ ) to a canonical sheaf morphism of algebras from $\mathrm{C}^{\infty}$ into $\mathcal{A}$. This sheaf morphism turns out to be a sheaf morphism of embeddings if $\Lambda$ is a directed set with respect to a partial order $\prec$ and if relation (7) holds.

We shall address the question of the embedding of $\mathcal{D}^{\prime}$ for the simple case of $\Lambda=(0,1]$. For a net $\left(\varphi_{\varepsilon}\right)_{\varepsilon}$ of mollifiers given by

$$
\varphi_{\varepsilon}(x)=\frac{1}{\varepsilon^{d}} \varphi\left(\frac{x}{\varepsilon}\right), x \in \mathbb{R}^{d} \text { where } \varphi \in \mathcal{D}\left(\mathbb{R}^{d}\right) \text { and } \int \varphi(x) d x=1,
$$

and $T \in \mathcal{D}^{\prime}\left(\mathbb{R}^{d}\right)$, the net $\left(T * \varphi_{\varepsilon}\right)_{\varepsilon}$ is a net of smooth functions in $\mathrm{C}^{\infty}\left(\mathbb{R}^{d}\right)$, moderately increasing in $\frac{1}{\varepsilon}$. This means that

$$
\forall K \Subset \mathbb{R}^{d}, \forall l \in N, \quad \exists m \in \mathbb{N}: p_{K, l}\left(T * \varphi_{\varepsilon}\right)=\mathrm{o}\left(\varepsilon^{-m}\right), \text { as } \varepsilon \rightarrow 0 .
$$

This justifies to choose

$$
\begin{aligned}
& A=\left\{\left(r_{\varepsilon}\right)_{\varepsilon} \in \mathbb{R}^{(0,1]}|\exists m \in \mathbb{N}:| u_{\varepsilon} \mid=\mathrm{o}\left(\varepsilon^{-m}\right), \text { as } \varepsilon \rightarrow 0\right\} \\
& I=\left\{\left(r_{\varepsilon}\right)_{\varepsilon} \in \mathbb{R}^{(0,1]}|\forall q \in \mathbb{N}:| u_{\varepsilon} \mid=\mathrm{o}\left(\varepsilon^{q}\right), \text { as } \varepsilon \rightarrow 0\right\} .
\end{aligned}
$$

In this case (with $\mathcal{E}=\mathrm{C}^{\infty}$ ), the sheaf of algebras $\mathcal{A}=\mathcal{H}_{(A, \mathcal{E}, \mathcal{P})} / \mathcal{J}_{\left(I_{A}, \mathcal{E}, \mathcal{P}\right)}$ is exactly the so-called special Colombeau algebra $\mathcal{G}$ [2, 7, 16]. Then, for all $\Omega \in \mathcal{O}\left(\mathbb{R}^{d}\right), \mathrm{C}^{\infty}(\Omega)$ is embedded in $\mathcal{A}(\Omega)$ by

$$
\sigma_{\Omega}: \mathrm{C}^{\infty}(\Omega) \rightarrow \mathcal{A}(\Omega) \quad f \mapsto\left[f_{\varepsilon}\right] \text { with } f_{\varepsilon}=f \text { for all } \varepsilon \text { in }(0,1],
$$

because the constant net $(f)_{\varepsilon}$ belongs to $\mathcal{H}_{(A, \mathcal{E}, \mathcal{P})}\left(\mathbb{R}^{d}\right)$ and $(f)_{\varepsilon} \in \mathcal{J}_{\left(I_{A}, \mathcal{E}, \mathcal{P}\right)}$ implies $f=0$ in $\mathrm{C}^{\infty}(\Omega)$. Furthermore, $\mathcal{D}^{\prime}\left(\mathbb{R}^{d}\right)$ is embedded in $\mathcal{A}\left(\mathbb{R}^{d}\right)$ by the mapping

$$
\iota: T \mapsto\left(T * \varphi_{\varepsilon}\right)_{\varepsilon}
$$

Indeed, relation (8) implies that $\left(T * \varphi_{\varepsilon}\right)_{\mathcal{\varepsilon}}$ belongs to $\mathcal{H}_{(A, \mathcal{E}, \mathcal{P})}\left(\mathbb{R}^{d}\right)$ and $\left(T * \varphi_{\varepsilon}\right)_{\mathcal{\varepsilon}} \in \mathcal{J}_{\left(I_{A}, \mathcal{E}, \mathcal{P}\right)}$ implies that $T * \varphi_{\varepsilon} \rightarrow 0$ in $\mathcal{D}^{\prime}\left(\mathbb{R}^{d}\right)$, as $\varepsilon \rightarrow 0$ and $T=0$. Thus, $\iota$ is a well defined injective map.

With the help of cutoff functions, we can define analogously, for each open set $\Omega$ in $\mathbb{R}^{d}$, an embedding $\iota_{\Omega}$ of $\mathcal{D}^{\prime}(\Omega)$ into $\mathcal{A}(\Omega)$, and finally a sheaf embedding $\mathcal{D}^{\prime} \rightarrow \mathcal{A}$. This embedding depends on the choice of the net of mollifiers $\left(\varphi_{\varepsilon}\right)_{\varepsilon}$. We refer the reader to [3, 15] for more complete discussions about embeddings in Colombeau's case and to 13 for the case of $(\mathcal{C}, \mathcal{E}, \mathcal{P})$ algebras.

\subsection{An association process}

We return to the general case with the assumption that $A$ is unitary and $\Lambda$ is a directed set with partial order relation $\prec$.

Let us denote by:

- $\Omega$ an open subset of $X$,

- $\mathcal{F}$ a given sheaf (or presheaf) of topological $\mathbb{K}$-vector spaces (resp. $\mathbb{K}$-algebras) over $X$ containing $\mathcal{E}$ as a subsheaf of topological algebras,

- $a$ a map from $\mathbb{R}_{+}$to $A_{+}$such that $a(0)=1$ (for $r \in \mathbb{R}_{+}$, we denote $a(r)$ by $\left.\left(a_{\lambda}(r)\right)_{\lambda}\right)$. 
In the Colombeau case, a typical example would be $a_{\varepsilon}(r)=\varepsilon^{r}$, $\varepsilon \in(0,1]$.

For $\left(v_{\lambda}\right)_{\lambda} \in \mathcal{H}_{(A, \mathcal{E}, \mathcal{P})}(\Omega)$, we shall denote the limit of $\left(v_{\lambda}\right)_{\lambda}$ for the $\mathcal{F}$-topology by $\lim _{\Lambda} \mathcal{F}(\Omega) v_{\lambda}$ when it exists. We recall that $\left.\lim _{\Lambda} \mathcal{F}(V) u_{\lambda}\right|_{V}=f \in \mathcal{F}(V)$ iff, for each $\mathcal{F}$-neighborhood $W$ of $f$, there exists $\lambda_{0} \in \Lambda$ such that

$$
\left.\lambda \prec \lambda_{0} \Longrightarrow u_{\lambda}\right|_{V} \in W .
$$

We suppose also that we have, for each open subset $V \subset \Omega$,

$$
\mathcal{J}_{\left(I_{A}, \mathcal{E}, \mathcal{P}\right)}(V) \subset\left\{\left(v_{\lambda}\right)_{\lambda} \in \mathcal{H}_{(A, \mathcal{E}, \mathcal{P})}(V): \lim _{\Lambda} \mathcal{F}(V) v_{\lambda}=0\right\}
$$

Definition 2 Consider $u=\left[u_{\lambda}\right] \in \mathcal{A}(\Omega), r \in \mathbb{R}_{+}, V$ an open subset of $\Omega$ and $f \in \mathcal{F}(V)$. We say that $u$ is a $(r)$-associated with $f$ in $V$ :

$$
u \underset{\mathcal{F}(V)}{\stackrel{a(r)}{\sim} f}
$$

if $\lim _{\Lambda} \mathcal{F}(V)\left(\left.a_{\lambda}(r) u_{\lambda}\right|_{V}\right)=f$.

In particular, if $r=0, u$ and $f$ are called associated in $V$.

To ensure the independence of the definition with respect to the representative of $u$, we must have, for any $\left(\eta_{\lambda}\right)_{\lambda} \in \mathcal{J}_{\left(I_{A}, \mathcal{E}, \mathcal{P}\right)}(\Omega)$, that $\left.\lim _{\Lambda} \mathcal{F}(V) a_{\lambda}(r) \eta_{\lambda}\right|_{V}=0$. As $\mathcal{J}_{\left(I_{A}, \mathcal{E}, \mathcal{P}\right)}(V)$ is a module over $A,\left(\left.a_{\lambda}(r) \eta_{\lambda}\right|_{V}\right)_{\lambda}$ is in $\mathcal{J}_{\left(I_{A}, \mathcal{E}, \mathcal{P}\right)}(V)$. Thus, our claim follows from hypothesis (9).

Example 1 Take $\left.\left.X=\mathbb{R}^{d}, \mathcal{F}=\mathcal{D}^{\prime}, \Lambda=\right] 0,1\right], \mathcal{A}=\mathcal{G}, V=\Omega, r=0$. The usual association between $u=\left[u_{\varepsilon}\right] \in \mathcal{G}(\Omega)$ and $T \in \mathcal{D}^{\prime}(\Omega)$ is defined by

$$
u \sim T \Longleftrightarrow u \underset{\mathcal{D}^{\prime}(\Omega)}{\stackrel{a(0)}{\sim}} T \Longleftrightarrow \lim _{\varepsilon \rightarrow 0} \mathcal{D}^{\prime}(\Omega) u_{\varepsilon}=T .
$$

\subsection{The $\mathcal{F}$-singular support of a generalized function}

We use the notations of Subsection 2.3. According to the hypothesis (9), we have, for any open set $\Omega$ in $X$,

$$
\mathcal{J}_{\left(I_{A}, \mathcal{E}, \mathcal{P}\right)}(\Omega) \subset\left\{\left(u_{\lambda}\right)_{\lambda} \in \mathcal{H}_{(A, \mathcal{E}, \mathcal{P})}(\Omega): \lim _{\Lambda} \mathcal{F}(V) u_{\lambda}=0\right\} .
$$

Set

$$
\mathcal{F}_{\mathcal{A}}(\Omega)=\left\{u \in \mathcal{A}(\Omega) \mid \exists\left(u_{\lambda}\right)_{\lambda} \in u, \exists f \in \mathcal{F}(\Omega): \lim _{\Lambda} \mathcal{F}(V) u_{\lambda}=f\right\} .
$$

$\mathcal{F}_{\mathcal{A}}(\Omega)$ is well defined because if $\left(\eta_{\lambda}\right)_{\lambda}$ belongs to $\mathcal{J}_{\left(I_{A}, \mathcal{E}, \mathcal{P}\right)}(\Omega)$, we have $\lim _{\Lambda} \mathcal{F}(V) \eta_{\lambda}=0$.

Moreover, $\mathcal{F}_{\mathcal{A}}$ is a sub-presheaf of vector spaces (resp. algebras) of $\mathcal{A}$. Roughly speaking, it is the presheaf whose sections above some open set $\Omega$ are the generalized functions of $\mathcal{A}(\Omega)$ associated with an element of $\mathcal{F}(\Omega)$.

Thus, for $u \in \mathcal{A}(\Omega)$, we can consider the set $\mathcal{O}_{\mathcal{A}}^{\mathcal{F}}(u)$ of all $x \in \Omega$ having an open neighborhood $V$ on which $u$ is associated with $f \in \mathcal{F}(V)$, that is:

$$
\mathcal{O}_{\mathcal{A}}^{\mathcal{F}}(u)=\left\{x \in \Omega\left|\exists V \in \mathcal{V}_{x}: u\right|_{V} \in \mathcal{F}_{\mathcal{A}}(V)\right\},
$$

$\mathcal{V}_{x}$ being the set of all the open neighborhoods of $x$.

This leads to the following definition: 
Definition 3 The $\mathcal{F}$-singular support of $u \in \mathcal{A}(\Omega)$ is denoted $\mathcal{S}_{\mathcal{A}}^{\mathcal{F}}(u)$ and defined as

$$
\mathcal{S}_{\mathcal{A}}^{\mathcal{F}}(u)=\Omega \backslash \mathcal{O}_{\mathcal{A}}^{\mathcal{F}}(u) .
$$

Remark 3 (i) The validity of the gluing principle $\left(F_{2}\right)$ is not necessary to get the notion of support (and of $\mathcal{F}$-singular support) of a section $u \in \mathcal{A}(\Omega)$. More precisely, the localization principle $\left(F_{1}\right)$ is sufficient to prove the following: The set

$$
\mathcal{O}_{\mathcal{A}}^{\{0\}}(u)=\left\{x \in \Omega\left|\exists V \in \mathcal{V}_{x}, u\right|_{V}=0\right\}
$$

is exactly the the union $\Omega_{\mathcal{A}}(u)$ of the open subsets of $\Omega$ on which $u$ vanishes.

Indeed, $\left(F_{1}\right)$ allows to show that $u$ vanishes on an open subset $\mathcal{O}$ of $\Omega$ if, and only if, it vanishes on an open neighborhood of every point of $\mathcal{O}$. This leads immediately to the required assertion. Moreover, $\Omega_{\mathcal{A}}(u)=\mathcal{O}_{\mathcal{A}}^{\{0\}}(u)$ is the largest open set on which u vanishes, $\mathcal{S}_{\mathcal{A}}^{\{0\}}(u)=\Omega \backslash \mathcal{O}_{\mathcal{A}}^{\{0\}}(u)$ is exactly the support of $u$ in its classical definition, and the $\mathcal{F}$-singular support of $u$ is a closed subset of its support.

(ii) In contrast to the situation described above for the support, we need the gluing principle $\left(F_{2}\right)$ if we want to prove that the restriction of $u$ to $\mathcal{O}_{\mathcal{A}}^{\mathcal{F}}(u)$ belongs to $\mathcal{F}_{\mathcal{A}}\left(\mathcal{O}_{\mathcal{A}}^{\mathcal{F}}(u)\right)$. We make this precise in the following lemma.

Lemma 4 Take $u \in \mathcal{A}(\Omega)$ and set $\Omega_{\mathcal{A}}^{\mathcal{F}}(u)=\cup_{i \in I} \Omega_{i},\left(\Omega_{i}\right)_{i \in I}$ denoting the collection of the open subsets of $\Omega$ such that $\left.u\right|_{\Omega_{i}} \in \mathcal{F}_{\mathcal{A}}\left(\Omega_{i}\right)$. Then, if $\mathcal{F}_{\mathcal{A}}$ is a sheaf (even if $\mathcal{A}$ is only a prehesaf),

(i) $\Omega_{\mathcal{A}}^{\mathcal{F}}(u)$ is the largest open subset $\mathcal{O}$ of $\Omega$ such that $\left.u\right|_{\mathcal{O}}$ belongs to $\mathcal{F}_{\mathcal{A}}(\mathcal{O})$;

(ii) $\Omega_{\mathcal{A}}^{\mathcal{F}}(u)=\mathcal{O}_{\mathcal{A}}^{\mathcal{F}}(u)$ and $\mathcal{S}_{\mathcal{A}}^{\mathcal{F}}(u)=\Omega \backslash \Omega_{\mathcal{A}}^{\mathcal{F}}(u)$.

Proof. (i) For $i \in I$, set $\left.u\right|_{\Omega_{i}}=f_{i} \in \mathcal{F}_{\mathcal{A}}\left(\Omega_{i}\right)$. The family $\left(f_{i}\right)_{i \in I}$ is coherent by assumption: From $\left(F_{2}\right)$, there exists $f \in \mathcal{F}_{\mathcal{A}}\left(\Omega_{\mathcal{A}}^{\mathcal{F}}(u)\right)$ such that $\left.f\right|_{\Omega_{i}}=f_{i}$. But from $\left(F_{1}\right)$, we have $f=u$ on $\cup_{i \in I} \Omega_{i}=\Omega_{\mathcal{A}}^{\mathcal{F}}(u)$. Thus $\left.u\right|_{\Omega_{\mathcal{A}}^{\mathcal{F}}(u)} \in \mathcal{F}_{\mathcal{A}}\left(\Omega_{\mathcal{A}}^{\mathcal{F}}(u)\right)$, and $\Omega_{\mathcal{A}}^{\mathcal{F}}(u)$ is clearly the largest open subset of $\Omega$ having this property.

(ii) First, $\mathcal{O}_{\mathcal{A}}^{\mathcal{F}}(u)$ is clearly an open subset of $\Omega$. For $x \in \mathcal{O}_{\mathcal{A}}^{\mathcal{F}}(u)$, set $\left.u\right|_{V_{x}}=f_{x} \in \mathcal{F}_{\mathcal{A}}\left(V_{x}\right)$ for some suitable neighborhood $V_{x}$. The open set $\mathcal{O}_{\mathcal{A}}^{\mathcal{F}}(u)$ can be covered by the family $\left(V_{x}\right)_{x \in \mathcal{O}_{\mathcal{A}}^{\mathcal{F}}(u)}$. As the family $\left(f_{x}\right)$ is coherent, we get from $\left(F_{2}\right)$ that there exists $f \in \mathcal{F}_{\mathcal{A}}\left(\cup_{x \in \mathcal{O}_{\mathcal{A}}^{\mathcal{F}}(u)} V_{x}\right)$ such that $\left.f\right|_{V_{x}}=f_{x}$. From $\left(F_{1}\right)$, we have $u=f$ on $\cup_{x \in \mathcal{O}_{\mathcal{A}}^{\mathcal{F}}(u)} V_{x}$ and, therefore, $\left.u\right|_{\mathcal{O}_{\mathcal{A}}^{\mathcal{F}}(u)} \in \mathcal{F}_{\mathcal{A}}\left(\mathcal{O}_{\mathcal{A}}^{\mathcal{F}}(u)\right)$. Thus $\mathcal{O}_{\mathcal{A}}^{\mathcal{F}}(u)$ is contained in $\Omega_{\mathcal{A}}^{\mathcal{F}}(u)$. Conversely, if $x \in \Omega_{\mathcal{A}}^{\mathcal{F}}(u)$, there exists an open neighborhood $V_{x}$ of $x$ such that $\left.u\right|_{V_{x}} \in \mathcal{F}_{\mathcal{A}}\left(V_{x}\right)$. Thus $x \in \mathcal{O}_{\mathcal{A}}^{\mathcal{F}}(u)$ and the assertion (ii) holds.

Proposition 5 For any $u, v \in \mathcal{A}(\Omega)$, if $\mathcal{F}$ is a presheaf of topological vector spaces, (resp. algebras), we have:

$$
\mathcal{S}_{\mathcal{A}}^{\mathcal{F}}(u+v) \subset \mathcal{S}_{\mathcal{A}}^{\mathcal{F}}(u) \cup \mathcal{S}_{\mathcal{A}}^{F}(v)
$$

Moreover, in the resp. case, we have

$$
\mathcal{S}_{\mathcal{A}}^{\mathcal{F}}(u v) \subset \mathcal{S}_{\mathcal{A}}^{F}(u) \cup \mathcal{S}_{\mathcal{A}}^{F}(v)
$$

Proof. If $x \in \Omega$ belongs to $\mathcal{O}_{\mathcal{A}}^{\mathcal{F}}(u) \cap \mathcal{O}_{\mathcal{A}}^{\mathcal{F}}(v)$, there exist $V$ and $W$ in $\mathcal{V}_{x}$ such that $\left.u\right|_{V} \in$ $\mathcal{F}_{\mathcal{A}}(V)$ and $\left.v\right|_{W} \in \mathcal{F}_{\mathcal{A}}(W)$. Thus $(u+v)_{\mid V \cap W} \in \mathcal{F}_{\mathcal{A}}(V \cap W)\left(\right.$ resp. $\left.(u v)_{\mid V \cap W} \in \mathcal{F}_{\mathcal{A}}(V \cap W)\right)$, which implies

$$
\mathcal{O}_{\mathcal{A}}^{\mathcal{F}}(u) \cap \mathcal{O}_{\mathcal{A}}^{\mathcal{F}}(v) \subset \mathcal{O}_{\mathcal{A}}^{\mathcal{F}}(u+v) \quad\left(\text { resp. } \mathcal{O}_{\mathcal{A}}^{\mathcal{F}}(u) \cap \mathcal{O}_{\mathcal{A}}^{\mathcal{F}}(v) \subset \mathcal{O}_{\mathcal{A}}^{\mathcal{F}}(u v)\right) .
$$

The result follows by taking the complementary sets in $\Omega$.

This proposition leads easily to the following: 
Corollary 6 Let $\left(u_{j}\right)_{1 \leq j \leq p}$ be any finite family of elements in $\mathcal{A}(\Omega)$. If $\mathcal{F}$ is a presheaf of topological vector spaces, (resp. algebras), we have

$$
\mathcal{S}_{\mathcal{A}}^{\mathcal{F}}\left(\sum_{1 \leq j \leq p} u_{j}\right) \subset \bigcup_{1 \leq j \leq p} \mathcal{S}_{\mathcal{A}}^{F}\left(u_{j}\right) .
$$

Moreover, in the resp. case, we have

$$
\mathcal{S}_{\mathcal{A}}^{\mathcal{F}}\left(\prod_{1 \leq j \leq p} u_{j}\right) \subset \bigcup_{1 \leq j \leq p} \mathcal{S}_{\mathcal{A}}^{F}\left(u_{j}\right)
$$

In particular, if $u_{j}=u$ for $1 \leq j \leq p$, we have $\mathcal{S}_{\mathcal{A}}^{\mathcal{F}}\left(u^{p}\right) \subset \mathcal{S}_{\mathcal{A}}^{F}(u)$.

Example 2 Taking $\mathcal{E}=\mathrm{C}^{\infty} ; \mathcal{F}=\mathcal{D}^{\prime} ; \mathcal{A}=\mathcal{G}$ leads to the $\mathcal{D}^{\prime}$-singular support of an element of the Colombeau algebra. This notion is complementary to the usual concept of local association in the Colombeau sense. We refer the reader to [12, 13] for more details.

Example 3 In the following examples we consider $X=\mathbb{R}^{d}, \mathcal{E}=\mathrm{C}^{\infty}$ and $\mathcal{A}=\mathcal{G}$.

(i) Take $u \in \sigma_{\Omega}\left(\mathrm{C}^{\infty}(\Omega)\right)$, where $\sigma_{\Omega}: \mathrm{C}^{\infty}(\Omega) \rightarrow \mathcal{G}(\Omega)$ is the canonical embedding defined in Subsection 2.9. Then $\mathcal{S}_{\mathcal{G}}^{\mathrm{C}^{p}}(u)=\varnothing$, for all $p \in \overline{\mathbb{N}}$.

(ii) Take $\varphi \in \mathcal{D}(\mathbb{R})$, with $\int \varphi(x) \mathrm{d} x=1$, and set $\varphi_{\varepsilon}(x)=\varepsilon^{-1} \varphi(x / \varepsilon)$. As $\varphi_{\varepsilon} \underset{\mathcal{D}^{\prime}(\mathbb{R})}{\stackrel{\varepsilon \rightarrow 0}{\longrightarrow}} \delta$, we have: $\mathcal{S}_{\mathcal{G}}^{\mathcal{D}^{\prime}}\left(\left[\varphi_{\varepsilon}\right]\right)=\{0\}$. We note also that $\mathcal{S}_{\mathcal{G}}^{C^{p}}\left(\left[\varphi_{\varepsilon}\right]\right)=\{0\}$. Indeed, for any $K \Subset \mathbb{R}^{*}=\mathbb{R} \backslash\{0\}$ and $\varepsilon$ small enough, $\varphi_{\varepsilon}$ is null on $K$ and, therefore, $\varphi_{\varepsilon} \underset{\mathrm{C}^{\infty}\left(\mathbb{R}^{*}\right)}{\stackrel{\varepsilon \rightarrow 0}{\longrightarrow}}=0$.

(iii) Take $u=\left[u_{\varepsilon}\right]$ with $u_{\varepsilon}(x)=\varepsilon \sin (x / \varepsilon)$. We have that $\lim p_{K, 0}\left(u_{\varepsilon}\right)=0$, for all $K \Subset \mathbb{R}$, whereas $\lim p_{K, 1}\left(u_{\varepsilon}\right)$ does not exist for $l \geq 1$. Therefore

$$
\mathcal{S}_{\mathcal{G}}^{\mathrm{C}^{0}}(u)=\varnothing, \quad \mathcal{S}_{\mathcal{G}}^{\mathrm{C}^{1}}(u)=\mathbb{R} .
$$

Remark 4 For any $(p, q) \in \overline{\mathbb{N}}^{2}$ with $p \leq q$, and $u \in \mathcal{G}$, it holds that $\mathcal{S}_{\mathcal{G}}^{\mathrm{C}^{p}}(u) \subset \mathcal{S}_{\mathcal{G}}^{\mathrm{C}^{q}}(u)$.

\section{The concept of $(a, \mathcal{F})$-microlocal analysis}

Let $\Omega$ be an open set in $X$. Fix $u=\left[u_{\lambda}\right] \in \mathcal{A}(\Omega)$ and $x \in \Omega$. The idea of the $(a, \mathcal{F})$-microlocal analysis is the following: $\left(u_{\lambda}\right)_{\lambda}$ may not tend to a section of $\mathcal{F}$ above a neighborhood of $x$, that is, there exists no $V \in \mathcal{V}_{x}$ and no $f \in \mathcal{F}(V)$ such that $\lim _{\Lambda} \mathcal{F}(V) u_{\lambda}=f$. Nevertheless, in this case, there may exist $V \in \mathcal{V}_{x}, r \geq 0$ and $f \in \mathcal{F}(V)$ such that $\lim _{\Lambda} \mathcal{F}(V) a_{\lambda}(r) u_{\lambda}=f$, that is $\left[\left.a_{\lambda}(r) u_{\lambda}\right|_{V}\right]$ belongs to the subspace (resp. subalgebra) $\mathcal{F}_{\mathcal{A}}(V)$ of $\mathcal{A}(V)$ introduced in Subsection 2.4. These preliminary remarks lead to the following concept.

\subsection{The $(a, \mathcal{F})$-singular parametric spectrum}

We recall that $a$ is a map from $\mathbb{R}_{+}$to $A_{+}$such that $a(0)=1$ and $\mathcal{F}$ is a presheaf of topological vector spaces (or topological algebras). For any open subset $\Omega$ of $X, u=\left[u_{\lambda}\right] \in \mathcal{A}(\Omega)$ and $x \in \Omega$, set

$$
\begin{aligned}
N_{(a, \mathcal{F}), x}(u) & =\left\{r \in \mathbb{R}_{+} \mid \exists V \in \mathcal{V}_{x}, \exists f \in \mathcal{F}(V): \lim _{\Lambda} \mathcal{F}(V)\right. \\
& =\left\{r \in \mathbb{R}_{+} \mid \exists V \in \mathcal{V}_{x}:\left[\left.a_{\lambda}(r) u_{\lambda}\right|_{V}\right)=f\right\} \\
& \left.\left.=\left.u_{\lambda}\right|_{V}\right] \in \mathcal{F}_{\mathcal{A}}(V)\right\} .
\end{aligned}
$$

It is easy to check that $N_{(a, \mathcal{F}), x}(u)$ does not depend on the representative of $u$. If no confusion may arise, we shall simply write

$$
N_{(a, \mathcal{F}), x}(u)=N_{x}(u)
$$


Theorem 7 Suppose that:

(a) For all $\lambda \in \Lambda$

$$
\forall(r, s) \in \mathbb{R}_{+}, \quad a_{\lambda}(r+s) \leq a_{\lambda}(r) a_{\lambda}(s),
$$

and, for all $r \in \mathbb{R}_{+} \backslash\{0\}$, the net $\left(a_{\lambda}(r)\right)_{\lambda}$ converges to 0 in $\mathbb{K}$.

(b) $\mathcal{F}$ is a presheaf of separated locally convex topological vector spaces.

Then we have, for $u \in \mathcal{A}(\Omega)$ :

(i) If $r \in N_{x}(u)$, then $[r,+\infty)$ is included in $N_{x}(u)$. Moreover, for all $s>r$, there exists $V \in \mathcal{V}_{x}$ such that: $\lim _{\Lambda} \mathcal{F ( V )}\left(\left.a_{\lambda}(s) u_{\lambda}\right|_{V}\right)=0$. Consequently, $N_{x}(u)$ is either empty, or a sub-interval of $\mathbb{R}_{+}$.

(ii) More precisely, suppose that for $x \in \Omega$, there exist $r \in \mathbb{R}_{+}, V \in \mathcal{V}_{x}$ and $f \in \mathcal{F}(V)$, nonzero on each neighborhood of $x$ included in $V$, such that $\lim _{\Lambda} \mathcal{F}(V)\left(\left.a_{\lambda}(r) u_{\lambda}\right|_{V}\right)=f$. Then $N_{x}(u)=[r,+\infty)$.

(iii) In the situation of $(i)$ and $(i i)$, we have that $0 \in N_{x}(u)$ iff $N_{x}(u)=\mathbb{R}_{+}$. Moreover, if one of these assertions holds, the limits $\lim _{\Lambda} \mathcal{F}(V)\left(\left.a_{\lambda}(s) u_{\lambda}\right|_{V}\right)$ can be non null only for $s=0$.

Proof. (i) If $r \in N_{x}(u)$, there exist $V \in \mathcal{V}_{x}$ and $f \in \mathcal{F}(V)$ such that $\lim _{\Lambda} \mathcal{F}(V)\left(a_{\lambda}(r) u_{\left.\lambda\right|_{V}}\right)=$ $f$. As $\mathcal{F}(V)$ is locally convex, its topology may be described by a family $\mathcal{Q}_{V}=\left(q_{j}\right)_{j \in J(V)}$ of semi-norms. For all $s>r$, we have, for any $j \in J(V)$,

$$
q_{j}\left(a_{\lambda}(s)\left(\left.u_{\lambda}\right|_{V}\right)\right)=a_{\lambda}(s) q_{j}\left(\left.u_{\lambda}\right|_{V}\right) \leq a_{\lambda}(s-r) a_{\lambda}(r) q_{j}\left(\left.u_{\lambda}\right|_{V}\right) \leq a_{\lambda}(s-r) q_{j}\left(\left.a_{\lambda}(r) u_{\lambda}\right|_{V}\right) .
$$

From $\lim _{\Lambda} q_{j}\left(a_{\lambda}(r)\left(\left.u_{\lambda}\right|_{V}-f\right)\right)=0$, we have $q_{j}\left(\left.a_{\lambda}(r) u_{\lambda}\right|_{V}\right)<+\infty$ and $\lim _{\Lambda} q_{j}\left(a_{\lambda}(s)\left(\left.u_{\lambda}\right|_{V}\right)\right)=$ 0 , since $a_{\lambda}(s-r) \stackrel{\Lambda}{\rightarrow} 0$. Thus $\lim _{\Lambda} \mathcal{F}(V)\left(\left.a_{\lambda}(s) u_{\lambda}\right|_{V}\right)=0$.

(ii) From $(i)$, we have $[r,+\infty) \subset N_{x}(u)$. Suppose that there exists $t<r$ in $N_{x}(u)$. Then we get $W \in \mathcal{V}_{x}$, which can be chosen included in $V$, and $g \in \mathcal{F}(W)$ such that $\lim _{\Lambda} \mathcal{F}(W)\left(\left.a_{\lambda}(t) u_{\lambda}\right|_{W}\right)=$ $g$. With the notations of the proof of $(i)$, we have

$$
q_{j}\left(a_{\lambda}(r)\left(\left.u_{\lambda}\right|_{W}\right)\right) \leq a_{\lambda}(r-t) q_{j}\left(\left.a_{\lambda}(t) u_{\lambda}\right|_{W}\right) .
$$

As $q_{j}\left(\left.a_{\lambda}(t) u_{\lambda}\right|_{V}\right)$ is bounded, it follows that $\lim _{\Lambda} q_{j}\left(a_{\lambda}(r)\left(\left.u_{\lambda}\right|_{W}\right)\right)=0$, which is in contradiction with $\lim _{\Lambda} \mathcal{F}(V)\left(a_{\lambda}(r)\left(\left.u_{\lambda}\right|_{V}\right)=f \not \equiv 0\right.$ on $W$.

(iii) The first assertion follows directly from $(i)$ and the second from (ii).

From now on, we suppose that the hypotheses $(a)$ and $(b)$ of Theorem 0 are fulfilled. We set

$$
\begin{aligned}
& \Sigma_{(a, \mathcal{F}), x}(u)=\Sigma_{x}(u)=\mathbb{R}_{+} \backslash N_{x}(u), \\
& R_{(a, \mathcal{F}), x}(u)=R_{x}(u)=\inf N_{x}(u) .
\end{aligned}
$$

According to the previous remarks and comments, $\Sigma_{(a, \mathcal{F}), x}(u)$ is an interval of $\mathbb{R}_{+}$of the form $\left[0, R_{(a, \mathcal{F}), x}(u)\right)$ or $\left[0, R_{(a, \mathcal{F}), x}(u)\right]$, the empty set, or $\mathbb{R}_{+}$.

Definition 4 The $(a, \mathcal{F})$-singular spectrum of $u \in \mathcal{A}(\Omega)$ is the set

$$
\mathcal{S}_{\mathcal{A}}^{(a, \mathcal{F})}(u)=\left\{(x, r) \in \Omega \times \mathbb{R}_{+} \mid r \in \Sigma_{x}(u)\right\} .
$$

Example 4 Take $X=\mathbb{R}^{d}, \mathcal{E}=\mathrm{C}^{\infty}, \mathcal{F}=\mathrm{C}^{p}(p \in \overline{\mathbb{N}}=\mathbb{N} \cup\{+\infty\}), f \in \mathrm{C}^{\infty}(\Omega)$. Set $u=$ $\left[\left(\varepsilon^{-1} f\right)_{\varepsilon}\right]$ and $v=\left[\left(\varepsilon^{-1}|\ln \varepsilon| f\right)_{\varepsilon}\right]$ in $\mathcal{A}(\Omega)=\mathcal{G}(\Omega)$. Then, for all $x \in \mathbb{R}$,

$$
N_{\left(a, \mathrm{C}^{p}\right), x}(u)=[1,+\infty), \quad N_{\left(a, \mathrm{C}^{p}\right), x}(v)=(1,+\infty), \quad R_{\left(a, \mathrm{C}^{p}\right), x}(u)=R_{\left(a, \mathrm{C}^{p}\right), x}(v)=1 .
$$


Remark 5 We have: $\Sigma_{(a, \mathcal{F}), x}(u)=\varnothing$ iff $N_{(a, \mathcal{F}), x}(u)=\mathbb{R}_{+}$and, according to Theorem $\emptyset$, iff $0 \in N_{(a, \mathcal{F}), x}(u)$, that is, there exist $(V, f) \in \mathcal{V}_{x} \times \mathcal{F}(V)$ such that $\lim _{\Lambda} \mathcal{F}(V)\left(\left.a_{\lambda}(0) u_{\lambda}\right|_{V}\right)=f . A s$ $a_{\lambda}(0) \equiv 1$, this last assertion is equivalent to $x \in \mathcal{O}_{\mathcal{A}}^{\mathcal{F}}(u)$. Thus $\Sigma_{(a, \mathcal{F}), x}(u)=\varnothing$ iff $x \notin \mathcal{S}_{\mathcal{A}}^{\mathcal{F}}(u)$.

This remark implies directly the:

Proposition 8 The projection of the $(a, \mathcal{F})$-singular spectrum of $u$ on $\Omega$ is the $\mathcal{F}$-singular support of $u$.

\subsection{Example: The Colombeau case}

In this subsection we investigate the relationship between the $(a, \mathcal{F})$-singular spectrum and the sharp topology for $X=\mathbb{R}^{d}, \mathcal{E}=\mathrm{C}^{\infty}, \mathcal{F}=\mathrm{C}^{p}(p \in \mathbb{N}), \mathcal{A}=\mathcal{G}, a_{\varepsilon}(r)=\varepsilon^{r}$. First, let us remark that, for $u=\left[u_{\varepsilon}\right] \in \mathcal{G}(\Omega), x \in \Omega\left(\Omega \in \mathcal{O}\left(\mathbb{R}^{d}\right)\right), N_{\left(a, \mathrm{C}^{p}\right), x}(u)$ is never empty.

Indeed, consider $V \in \mathcal{V}_{x}$ with $\bar{V} \Subset \Omega$. There exists $m>0$ such that $p_{p, \bar{V}}\left(u_{\varepsilon}\right)=\mathrm{o}\left(\varepsilon^{-m}\right)$ as $\varepsilon \rightarrow 0$. Thus, $p_{k, \bar{V}}\left(u_{\varepsilon}\right)=o\left(\varepsilon^{-m}\right)$ for all $k \leq p$ and $\lim _{\varepsilon \rightarrow 0} \mathrm{C}^{p}(V)\left(\left.\varepsilon^{m} u_{\varepsilon}\right|_{V}\right)=0$. Thus $[m,+\infty) \subset$ $N_{\left(a, \mathrm{C}^{p}\right), x}(u)$.

Let us now recall the construction of the sharp topology on $\mathcal{G}(\Omega)$. For $u=\left[\left(u_{\varepsilon}\right)_{\varepsilon}\right] \in \mathcal{G}(\Omega)$, $K \Subset \Omega, l \in \mathbb{N}$, set

$$
v_{K, l}(u)=\inf \left\{r \in \mathbb{R} \mid p_{K, l}\left(u_{\varepsilon}\right)=\mathrm{o}\left(\varepsilon^{-r}\right) \text { as } \varepsilon \rightarrow 0\right\}
$$

The real number $v_{K, l}(u)$ is well defined, i.e. does not depend on the representative of $u$, and is called the $(K, l)$-valuation of $u$. It has the usual properties:

(i) $\forall \lambda \in \mathbb{C} \backslash\{0\}, \forall u \in \mathcal{G}(\Omega), v_{K, l}(\lambda u)=v_{K, l}(u)$;

(ii) $\forall u, v \in \mathcal{G}(\Omega), v_{K, l}(u+v) \leq \sup \left(v_{K, l}(u), v_{K, l}(v)\right)$.

The family $\left(v_{K, l}\right)$ permits to define the $(K, l)$-pseudodistances $d_{K, l}$ on $\mathcal{G}(\Omega)$ by

$$
\forall(u, v) \in \mathcal{G}(\Omega)^{2}, \quad d_{K, l}(u, v)=\exp \left(v_{K, l}(u-v)\right),
$$

which turns out to be ultrametric:

$$
\forall(u, v, w) \in \mathcal{G}(\Omega)^{3}, \quad d_{K, l}(u, v) \leq \sup \left(d_{K, l}(u, w), d_{K, l}(w, v)\right) .
$$

The topology defined by the family $\left(d_{K, l}\right)_{K, l}$ is called the sharp topology on $\mathcal{G}(\Omega)$.

As we are interested here in valuations greater or equal to 0 , we set, for $u \in \mathcal{G}(\Omega)$,

$$
\nu_{K, l}(u)=\sup \left(v_{K, l}(u), 0\right) .
$$

We can define, for $x \in \Omega$, the l-valuation of $u$ at $x$ by

$$
\nu_{x, l}(u)=\inf \left\{\nu_{\bar{V}, l}(u) \mid V \in \mathcal{V}(x), V \text { relatively compact }\right\}
$$

and set, for any $p \in \overline{\mathbb{N}}$,

$$
\nu_{x}^{p}(u)=\sup _{0 \leq l \leq p} \nu_{x, l}(u)
$$

Proposition 9 For all $p \in \overline{\mathbb{N}},\left[u_{\varepsilon}\right] \in \mathcal{G}(\Omega)$ and $x \in \Omega$, we have

$$
\nu_{x}^{p}(u)=R_{\left(a, \mathrm{C}^{p}\right), x}(u)=\inf N_{\left(a, \mathrm{C}^{p}\right), x}(u) .
$$

Proof. Take $r>\nu_{x}^{p}(u)$. Then, for any $l$ with $0 \leq l \leq p$, one has $r>\nu_{x, l}(u)$ and there exists $V \in \mathcal{V}(x), V$ relatively compact, such that $v_{\bar{V}, l}(u)<r$. Thus, $p_{\bar{V}, l}\left(u_{\varepsilon}\right)=\mathrm{o}\left(\varepsilon^{-r}\right)$, as $\varepsilon \rightarrow 0$, and $\lim _{\varepsilon \rightarrow 0} \mathrm{C}^{p}(V)\left(\left.\varepsilon^{r} u_{\varepsilon}\right|_{V}\right)=0$, which implies that $r>R_{\left(a, \mathrm{C}^{p}\right), x}(u)$ and $\nu_{x}^{p}(u) \geq R_{\left(a, \mathrm{C}^{p}\right), x}(u)$. Conversely, if $r>R_{\left(a, \mathrm{C}^{p}\right), x}(u)$, there exists $V \in \mathcal{V}(x)$ such that $\left.\lim _{\varepsilon \rightarrow 0} \mathrm{C}^{p}(V)=\left.\varepsilon^{r} u_{\varepsilon}\right|_{V}\right)=0$. For any relatively compact neighborhood $W$ of $x$ included in $V$, we get $p_{\bar{W}, l}\left(u_{\varepsilon}\right)=\mathrm{o}\left(\varepsilon^{-r}\right)$ and $r>v_{\bar{W}, l}(u)>\nu_{x, l}(u)$. Thus, $r \geq \nu_{x}^{p}(u)$ and $\nu_{x}^{p}(u) \leq R_{\left(a, \mathrm{C}^{p}\right), x}(u)$. 


\subsection{Some properties of the $(a, \mathcal{F})$-singular parametric spectrum}

Notation 2 For $u=\left[u_{\lambda}\right] \in \mathcal{A}(\Omega), \lim _{\Lambda} \mathcal{F ( V )}\left(\left.a_{\lambda}(r) u_{\lambda}\right|_{V}\right) \in \mathcal{F}(V)$ means that there exists $f \in \mathcal{F}(V)$ such that $\lim _{\Lambda} \mathcal{F}(V)\left(\left.a_{\lambda}(r) u_{\lambda}\right|_{V}\right)=f$.

\subsubsection{Linear properties}

Proposition 10 For any $u, v \in \mathcal{A}(\Omega)$, we have

$$
\mathcal{S}_{\mathcal{A}}^{(a, \mathcal{F})}(u+v) \subset \mathcal{S}_{\mathcal{A}}^{(a, \mathcal{F})}(u) \cup \mathcal{S}_{\mathcal{A}}^{(a, \mathcal{F})}(v) .
$$

Proof. Let $r$ be in $N_{x}(u) \cap N_{x}(v)$. Then there exist $V \in \mathcal{V}_{x}$ and $W \in \mathcal{V}_{x}$ such that

$$
\lim _{\Lambda} \mathcal{F}(V)\left(\left.a_{\lambda}(r) u_{\lambda}\right|_{V}\right) \in \mathcal{F}(V) \text { and } \lim _{\Lambda} \mathcal{F}(W)\left(\left.a_{\lambda}(r) v_{\lambda}\right|_{W}\right) \in \mathcal{F}(W) .
$$

Thus $\lim _{\Lambda} \mathcal{F}(V \cap W)\left(\left.a_{\lambda}(r)\left(u_{\lambda}+v_{\lambda}\right)\right|_{V \cap W}\right) \in \mathcal{F}(V \cap W)$ and $r \in N_{x}(u+v)$. Consequently,

$$
N_{x}(u) \cap N_{x}(v) \subset N_{x}(u+v) .
$$

We obtain the result by taking the complementary sets in $\mathbb{R}_{+}$.

Corollary 11 For any $u, u_{0}, u_{1}$ in $\mathcal{A}(\Omega)$ with

$$
\text { (i) } u=u_{0}+u_{1} \quad(\text { ii }) \mathcal{S}_{\mathcal{A}}^{(a, \mathcal{F})}\left(u_{0}\right)=\varnothing,
$$

we have

$$
\mathcal{S}_{\mathcal{A}}^{(a, \mathcal{F})}(u)=\mathcal{S}_{\mathcal{A}}^{(a, \mathcal{F})}\left(u_{1}\right)
$$

Proof. Proposition 10 and condition $(i i)$ give $\mathcal{S}_{\mathcal{A}}^{(a, \mathcal{F})}(u) \subset \mathcal{S}_{\mathcal{A}}^{(a, \mathcal{F})}\left(u_{1}\right)$. As $(i)$ implies $u_{0}=u-u_{1}$, we obtain the converse inclusion, and thus the equality.

\subsubsection{Differential properties}

We suppose that $\mathcal{F}$ is a sheaf of topological differential vector spaces (resp. algebras), with continuous differentiation, admitting $\mathcal{E}$ as a subsheaf of topological differential algebras. Then the sheaf $\mathcal{A}$ is also a sheaf of differential algebras with, for any $\alpha \in \mathbb{N}^{d}$ and $u \in \mathcal{A}(\Omega)$,

$$
\partial^{\alpha} u=\left[\partial^{\alpha} u_{\lambda}\right] \text {, where }\left(u_{\lambda}\right)_{\lambda} \text { is any representative of } u \text {. }
$$

The independence of $\partial^{\alpha} u$ on the choice of representative follows directly from the definition of $\left.\mathcal{J}_{\left(I_{A}, \mathcal{E}, \mathcal{P}\right)} \cdot\right)$

Proposition 12 Let $u$ be in $\mathcal{A}(\Omega)$. For all $\partial^{\alpha}, \alpha \in \mathbb{N}^{d}$, we have

$$
\mathcal{S}_{\mathcal{A}}^{(a, \mathcal{F})}\left(\partial^{\alpha} u\right) \subset \mathcal{S}_{\mathcal{A}}^{(a, \mathcal{F})}(u) .
$$

Proof. Take $u \in \mathcal{A}(\Omega), \alpha \in \mathbb{N}^{d}, x \in \Omega, r \in N_{x}(u)$. There exists $V \in \mathcal{V}_{x}, f \in \mathcal{F}(V)$ such that

$$
\lim _{\Lambda} \mathcal{F ( V )}\left(\left.a_{\lambda}(r) u_{\lambda}\right|_{V}\right)=f
$$

The continuity of $\partial^{\alpha}$ implies that

$$
\lim _{\Lambda} \mathcal{F ( V )}\left(\left.a_{\lambda}(r) \partial^{\alpha} u_{\lambda}\right|_{V}\right)=\partial^{\alpha} f
$$

Thus $N_{x}(u) \subset N_{x}\left(\partial^{\alpha} u\right)$. The result is proved.

In the following two results we require that $\mathcal{F}$ is a sheaf of topological modules over $\mathcal{E}$, in addition. The proofs are straightforward. 
Proposition 13 Let $g$ be in $\mathcal{E}(\Omega)$ and $u$ in $\mathcal{A}(\Omega)$. We have

$$
\mathcal{S}_{\mathcal{A}}^{(a, \mathcal{F})}(g u) \subset \mathcal{S}_{\mathcal{A}}^{(a, \mathcal{F})}(u)
$$

Propositions 10, 12 and 13 finally imply:

Corollary 14 Let $P(\partial)=\sum_{|\alpha| \leq m} C_{\alpha} \partial^{\alpha}$ be a differential polynomial with coefficients in $\mathcal{E}(\Omega)$. For any $u \in \mathcal{A}(\Omega)$, we have

$$
\mathcal{S}_{\mathcal{A}}^{(a, \mathcal{F})}(P(\partial) u) \subset \mathcal{S}_{\mathcal{A}}^{(a, \mathcal{F})}(u)
$$

\subsubsection{Nonlinear properties}

Theorem 15 For given $u$ and $v \in \mathcal{A}(\Omega)$, let $D_{i}(i=1,2,3)$ be the following disjoint sets:

$$
D_{1}=\mathcal{S}_{\mathcal{A}}^{\mathcal{F}}(u) \backslash\left(\mathcal{S}_{\mathcal{A}}^{\mathcal{F}}(u) \cap \mathcal{S}_{\mathcal{A}}^{\mathcal{F}}(v)\right) ; \quad D_{2}=\mathcal{S}_{\mathcal{A}}^{\mathcal{F}}(v) \backslash\left(\mathcal{S}_{\mathcal{A}}^{\mathcal{F}}(u) \cap \mathcal{S}_{\mathcal{A}}^{\mathcal{F}}(v)\right) ; \quad D_{3}=\mathcal{S}_{\mathcal{A}}^{\mathcal{F}}(u) \cap \mathcal{S}_{\mathcal{A}}^{\mathcal{F}}(v) .
$$

Then the $(a, \mathcal{F})$-singular asymptotic spectrum of uv verifies

$$
\mathcal{S}_{\mathcal{A}}^{(a, \mathcal{F})}(u v) \subset\left\{\left(x, \Sigma_{x}(u)\right), x \in D_{1}\right\} \cup\left\{\left(x, \Sigma_{x}(v)\right), x \in D_{2}\right\} \cup\left\{\left(x, E_{x}(u, v)\right), x \in D_{3}\right\}
$$

where (for any $x \in D_{3}$ )

$$
E_{x}(u, v)=\left\{\begin{array}{l}
{\left[0, \sup \Sigma_{x}(u)+\sup \Sigma_{x}(v)\right] \text { if } \Sigma_{x}(u) \neq \mathbb{R}_{+} \text {and } \Sigma_{x}(v) \neq \mathbb{R}_{+}} \\
\mathbb{R}_{+} \text {if } \Sigma_{x}(u)=\mathbb{R}_{+} \text {or } \Sigma_{x}(v)=\mathbb{R}_{+}
\end{array}\right.
$$

Proof. Suppose that $x$ belongs to $D_{1}$. Then $x$ is not in $\mathcal{S}_{\mathcal{A}}^{\mathcal{F}}(v)$ and we have

$$
\Sigma_{x}(v)=\varnothing, N_{x}(v)=\mathbb{R}_{+} .
$$

If $N_{x}(u)$ is not empty, let $r$ be in $N_{x}(u)$. As $N_{x}(v)=\mathbb{R}_{+}$, we have $r \in N_{x}(v)$. Thus there exists $V \in \mathcal{V}_{x}\left(\operatorname{resp} . W \in \mathcal{V}_{x}\right)$ such that $\left[\left.a_{\lambda}(r) u_{\lambda}\right|_{V}\right] \in \mathcal{F}_{\mathcal{A}}(V)\left(\operatorname{resp} .\left[\left.a_{\lambda}(r) v_{\lambda}\right|_{W}\right] \in \mathcal{F}_{\mathcal{A}}(W)\right)$. As $\mathcal{F}$ is a sheaf of topological algebras we have

$$
\left[\left.a_{\lambda}(r)\left(u_{\lambda} v_{\lambda}\right)\right|_{V \cap W}\right] \in \mathcal{F}_{\mathcal{A}}(V \cap W) .
$$

Thus, $r$ belongs to $N_{x}(u v)$. Therefore, we have proved that $\Sigma_{x}(u v) \subset \Sigma_{x}(u)$. If $N_{x}(u)$ is empty, we have $\Sigma_{x}(u)=\mathbb{R}_{+}$and the above inclusion is obviously fulfilled. For $x$ in $D_{2}$, the same proof gives $\Sigma_{x}(u v) \subset \Sigma_{x}(v)$.

Consider $x$ in $D_{3}$. Then, $\Sigma_{x}(u)$ and $\Sigma_{x}(v)$ are not empty. We suppose first that both of them are not equal to $\mathbb{R}_{+}$. Set $R=\sup \Sigma_{x}(u)$ and $S=\sup \Sigma_{x}(v)$. If $r>R$, there exists $r^{\prime} \in N_{x}(u)$ such that $R<r^{\prime}<r$ and then, from the part $(i)$ of Theorem 0 , there exists $V \in \mathcal{V}_{x}$ such that

$$
\lim _{\Lambda} \mathcal{F}(V)\left(\left.a_{\lambda}(r) u_{\lambda}\right|_{V}\right)=0
$$

Similarly, if $s>S$, there exists $W \in \mathcal{V}_{x}$ such that

$$
\lim _{\Lambda} \mathcal{F}(W)\left(\left.a_{\lambda}(s) v_{\lambda}\right|_{W}\right)=0 .
$$

Then $\lim _{\Lambda} \mathcal{F}(V \cap W)\left(\left.a_{\lambda}(r) a_{\lambda}(s)\left(u_{\lambda} v_{\lambda}\right)\right|_{V \cap W}\right)=0$. By expressing this limit in terms of seminorms, as in the proof of Theorem 7 and by using the inequality $a_{\lambda}(r+s) \leq a_{\lambda}(r) a_{\lambda}(s)$, we get that $\lim _{\Lambda} \mathcal{F ( V \cap W )}\left(\left.a_{\lambda}(r+s)\left(u_{\lambda} v_{\lambda}\right)\right|_{V \cap W}\right)=0$. Thus

$$
\left[r+s, \infty\left[\subset N _ { x } ( u v ) \text { or } \left[0, r+s\left[\supset \Sigma_{x}(u v)\right.\right.\right.\right.
$$

for any $r>R$ and $s>S$. Thus

$$
\Sigma_{x}(u v) \subset[0, R+S]=\left[0, \sup \Sigma_{x}(u)+\sup \Sigma_{x}(v)\right] .
$$

If $\Sigma_{x}(u)$ or $\Sigma_{x}(v)$ is equal to $\mathbb{R}_{+}$, the obvious inclusion $\Sigma_{x}(u v) \subset \mathbb{R}_{+}$gives the last result. 
Corollary 16 For given $u \in \mathcal{A}(\Omega)$ and $p \in \mathbb{N}^{*}$, we have

$$
\mathcal{S}_{\mathcal{A}}^{(a, \mathcal{F})}\left(u^{p}\right) \subset\left\{\left(x, H_{p, x}(u)\right), x \in \mathcal{S}_{\mathcal{A}}^{\mathcal{F}}(u)\right\} .
$$

where $H_{p, x}(u)=\left\{\begin{array}{l}{\left[0, p \sup \Sigma_{x}(u)\right] \text { if } \Sigma_{x}(u) \neq \mathbb{R}_{+}} \\ \mathbb{R}_{+} \text {if } \Sigma_{x}(u)=\mathbb{R}_{+}\end{array}\right.$

Proof. When $\Sigma_{x}(u)=\mathbb{R}_{+}$, the result is obvious. Suppose now $\Sigma_{x}(u) \neq \mathbb{R}_{+}$. We shall prove the result by induction. If $p=1$, the result is a simple consequence of the definitions. Suppose that the result holds for some $p \geq 1$. Set $v=u^{p}$ in the previous theorem. We have

$$
D_{1}=\mathcal{S}_{\mathcal{A}}^{\mathcal{F}}(u) \backslash \mathcal{S}_{\mathcal{A}}^{\mathcal{F}}\left(u^{p}\right) ; \quad D_{2}=\varnothing ; \quad D_{3}=\mathcal{S}_{\mathcal{A}}^{\mathcal{F}}\left(u^{p}\right) .
$$

Thus

$$
\mathcal{S}_{\mathcal{A}}^{(a, \mathcal{F})}\left(u^{p+1}\right) \subset\left\{\left(x, \Sigma_{x}(u)\right), x \in \mathcal{S}_{\mathcal{A}}^{\mathcal{F}}(u) \backslash \mathcal{S}_{\mathcal{A}}^{\mathcal{F}}\left(u^{p}\right)\right\} \cup\left\{\left(x,\left[0,(p+1) \sup \Sigma_{x}(u)\right]\right), x \in \mathcal{S}_{\mathcal{A}}^{\mathcal{F}}\left(u^{p}\right)\right\},
$$

by using the induction hypothesis. It follows a fortiori that

$$
\mathcal{S}_{\mathcal{A}}^{(a, \mathcal{F})}\left(u^{p+1}\right) \subset\left\{\left(x,\left[0,(p+1) \sup \Sigma_{x}(u)\right]\right), x \in \mathcal{S}_{\mathcal{A}}^{\mathcal{F}}(u)\right\}
$$

\section{Applications to partial differential equations}

In this section we shall compute various $(a, \mathcal{F})$-singular spectra of solutions to linear and nonlinear partial differential equations. Throughout we shall suppose that $\Lambda=] 0,1], X=\mathbb{R}^{d}, \mathcal{E}=\mathrm{C}^{\infty}$, $\mathcal{F}=\mathrm{C}^{p}(1 \leq p \leq \infty)$ or $\mathcal{F}=\mathcal{D}^{\prime}, a_{\varepsilon}(r)=\varepsilon^{r}$. The results will hold for any $(\mathcal{C}, \mathcal{E}, \mathcal{P})$-algebra

$$
\mathcal{A}=\mathcal{H}_{(A, \mathcal{E}, \mathcal{P})} / \mathcal{J}_{\left(I_{A}, \mathcal{E}, \mathcal{P}\right)}
$$

such that $\left(a_{\varepsilon}(r)\right)_{\varepsilon} \in A_{+}$for all $r \in \mathbb{R}_{+}$and property (9) holds.

Example 5 The $\left(a, \mathrm{C}^{p}\right)$-singular spectrum of powers of the delta function. Given a mollifier of the form

$$
\varphi_{\varepsilon}(x)=\frac{1}{\varepsilon^{d}} \varphi\left(\frac{x}{\varepsilon}\right), x \in \mathbb{R}^{d} \text { where } \varphi \in \mathcal{D}\left(\mathbb{R}^{d}\right), \varphi \geq 0 \text { and } \int \varphi(x) d x=1,
$$

its class in $\mathcal{A}\left(\mathbb{R}^{d}\right)$ defines the delta function $\delta(x)$ as an element of $\mathcal{A}\left(\mathbb{R}^{d}\right)$. Its powers are given by $(m \in \mathbb{N})$

$$
\delta^{m}=\left[\varphi_{\varepsilon}^{m}\right]=\left[\frac{1}{\varepsilon^{m d}} \varphi^{m}\left(\frac{\dot{\varepsilon}}{\varepsilon}\right)\right] .
$$

Clearly, the $\mathrm{C}^{0}$-singular spectrum is given by

$$
\mathcal{S}_{\mathcal{A}}^{\left(a, \mathrm{C}^{0}\right)}\left(\delta^{m}\right)=(0,[0, m d]) .
$$

Differentiating $\varphi^{m}(x)$ and observing that for each derivative there is a point $x$ at which it does not vanish we see that

$$
\mathcal{S}_{\mathcal{A}}^{\left(a, \mathrm{C}^{k}\right)}\left(\delta^{m}\right)=(0,[0, m d+k]) .
$$

Example 6 The $\left(a, \mathcal{D}^{\prime}\right)$-singular spectrum of powers of the delta function. Given a test function $\psi \in \mathcal{D}\left(\mathbb{R}^{d}\right)$, we have

$$
\int \varphi_{\varepsilon}^{m}(x) \psi(x) d x=\int \frac{1}{\varepsilon^{m d-d}} \varphi^{m}(x) \psi(\varepsilon x) d x
$$

thus

$$
\mathcal{S}_{\mathcal{A}}^{\left(a, \mathcal{D}^{\prime}\right)}\left(\delta^{m}\right)=\varnothing \text { for } m=1, \quad \mathcal{S}_{\mathcal{A}}^{\left(a, \mathcal{D}^{\prime}\right)}\left(\delta^{m}\right)=(0,[0, m d-d[) \text { for } m>1 .
$$




\subsection{The singular spectrum of solutions to linear hyperbolic equations}

Consider the Cauchy problem for the $d$-dimensional linear wave equation

$$
\begin{aligned}
& \partial_{t}^{2} u_{\varepsilon}(x, t)-\Delta u_{\varepsilon}(x, t)=0, \quad x \in \mathbb{R}^{d}, t \in \mathbb{R} \\
& u_{\varepsilon}(x, 0)=u_{0 \varepsilon}(x), \partial_{t} u_{\varepsilon}(x, 0)=u_{1 \varepsilon(x)}, \quad x \in \mathbb{R}^{d},
\end{aligned}
$$

where $u_{0 \varepsilon}, u_{1 \varepsilon} \in \mathrm{C}^{\infty}\left(\mathbb{R}^{d}\right)$ represent elements $u_{0}, u_{1}$ of an algebra $\mathcal{A}\left(\mathbb{R}^{d}\right)$ as outlined at the beginning of this section. Under suitable assumptions on the ring $A$, the corresponding net of classical smooth solutions represents a unique solution $u$ in the algebra $\mathcal{A}\left(\mathbb{R}^{d+1}\right)$; for example, this holds in the Colombeau case [16]. Let $t \rightarrow E(t) \in \mathrm{C}^{\infty}\left(\mathbb{R}: \mathcal{E}^{\prime}\left(\mathbb{R}^{d}\right)\right)$ be the fundamental solution of the Cauchy problem. Then

$$
u_{\varepsilon}(\cdot, t)=\frac{d}{d t} E(t) * \varepsilon^{r} u_{0 \varepsilon}+E(t) * \varepsilon^{r} u_{1 \varepsilon} .
$$

If for some $r \geq 0$ and $u_{0} \in \mathcal{D}^{\prime}\left(\mathbb{R}^{d}\right)$,

$$
\int \varepsilon^{r} u_{0 \varepsilon}(x) \psi(x) d x \rightarrow\left\langle u_{0}, \psi\right\rangle
$$

for all $\psi \in \mathcal{D}\left(\mathbb{R}^{d}\right)$, then

$$
\int \varepsilon^{r} u_{\varepsilon}(x) \psi(x) d x=\left\langle E(t) * \varepsilon^{r} u_{0 \varepsilon}, \psi\right\rangle=\left\langle\varepsilon^{r} u_{0 \varepsilon}, \check{E}(t) * \psi\right\rangle \rightarrow\left\langle u_{0}, \check{E}(t) * \psi\right\rangle
$$

for all $\psi \in \mathcal{D}\left(\mathbb{R}^{d}\right)$ and $t \in \mathbb{R}$ as well. We arrive at the following assertion.

Proposition 17 Assume that $\mathcal{S}_{\mathcal{A}}^{\left(a, \mathcal{D}^{\prime}\right)}\left(u_{0}\right)$ and $\mathcal{S}_{\mathcal{A}}^{\left(a, \mathcal{D}^{\prime}\right)}\left(u_{1}\right)$ are contained in $\mathbb{R}^{d} \times I$, where $I=\varnothing$, $I=\left[0, r\left[\right.\right.$ or $I=[0, r]$ for some $r, 0 \leq r \leq \infty$. Let $u \in \mathcal{A}\left(\mathbb{R}^{d+1}\right)$ be the solution to the linear wave equation (10). Then $\mathcal{S}_{\mathcal{A}}^{\left(a, \mathcal{D}^{\prime}\right)}(u(\cdot, t)) \subset \mathbb{R}^{d} \times I$ for all $t \in \mathbb{R}$.

This upper bound may or may not be reached, depending on the effects of finite propagation speed or the Huyghens principle in odd space dimension $d \geq 3$. We just illustrate some of the possible effects for the one-dimensional wave equation with powers of delta functions as initial data. Thus we consider the problem

$$
\begin{aligned}
& \partial_{t}^{2} u_{\varepsilon}(x, t)-\partial_{x}^{2} u_{\varepsilon}(x, t)=0, \quad x \in \mathbb{R}, t \in \mathbb{R} \\
& u_{\varepsilon}(x, 0)=c_{0} \varphi_{\varepsilon}^{m}(x), \partial_{t} u_{\varepsilon}(x, 0)=c_{1} \varphi_{\varepsilon}^{n}(x), \quad x \in \mathbb{R},
\end{aligned}
$$

where $\varphi$ is a mollifier as in Example 5 and $c_{0}, c_{1} \in \mathbb{R}$. The solution to (11) is given by

$$
u_{\varepsilon}(x, t)=\frac{c_{0}}{2}\left(\varphi_{\varepsilon}^{m}(x-t)+\varphi_{\varepsilon}^{m}(x+t)\right)+\frac{c_{1}}{2} \int_{x-t}^{x+t} \varphi_{\varepsilon}^{n}(y) d y .
$$

We observe that $u_{\varepsilon}(x, t)=0$ for sufficiently small $\varepsilon$ when $|x|>|t|$, that is, outside the light cone, and $u_{\varepsilon}(x, t)=\operatorname{sign}(t) \frac{c_{1}}{2} \varepsilon^{n-1}\left\|\varphi^{n}\right\|_{\mathrm{L}^{1}(\mathbb{R})}$ for sufficiently small $\varepsilon$ when $|x|<|t|$.

Example 7 If in equation (11) $c_{0} \neq 0, c_{1}=0$ then

$$
\mathcal{S}_{\mathcal{A}}^{\left(a, \mathcal{D}^{\prime}\right)}(u)=\{(x, t, r):|x|=|t|, 0 \leq r<m-1\}
$$

with the provision that $\mathcal{S}_{\mathcal{A}}^{\left(a, \mathcal{D}^{\prime}\right)}(u)=\varnothing$ when $m=1$. If in equation (11) $c_{0}=0, c_{1} \neq 0$ then

$$
\mathcal{S}_{\mathcal{A}}^{\left(a, \mathcal{D}^{\prime}\right)}(u)=\{(x, t, r):|x| \leq|t|, 0 \leq r<n-1\} .
$$


When both $c_{0}$ and $c_{1}$ are nonzero the singular spectrum is obtained as the union of the two spectra. For the $\mathrm{C}^{0}$-singular spectrum the following results hold: If in equation (11) $c_{0} \neq 0$, $c_{1}=0$ then

$$
\mathcal{S}_{\mathcal{A}}^{\left(a, \mathrm{C}^{0}\right)}(u)=\{(x, t, r):|x|=|t|, 0 \leq r \leq m\} .
$$

If $c_{0}=0, c_{1} \neq 0$ then

$$
\mathcal{S}_{\mathcal{A}}^{\left.\left(a, \mathrm{C}^{0}\right)\right)}(u)=\{(x, t, r):|x|<|t|, 0 \leq r<n-1\} \cup\{(x, t, r):|x|=|t|, 0 \leq r \leq n-1\} .
$$

\subsection{The singular spectrum of solutions to semilinear hyperbolic equations}

In this subsection we study the paradigmatic case of a semilinear transport equation

$$
\begin{aligned}
& \partial_{t} u_{\varepsilon}(x, t)+\lambda(x, t) \partial_{x} u_{\varepsilon}(x, t)=F\left(u_{\varepsilon}(x, t)\right), \quad x \in \mathbb{R}, t \in \mathbb{R} \\
& u_{\varepsilon}(x, 0)=u_{0 \varepsilon}(x), \quad x \in \mathbb{R}
\end{aligned}
$$

where $\lambda$ and $F$ are smooth functions of their arguments. In this situation, the singular spectrum of the initial data may be decreased or increased, depending on the function $F$. We observe that by a change of coordinates we may assume without loss of generality that $\lambda \equiv 0$. In fact, denote by $s \rightarrow \gamma(x, t, s)$ the characteristic curve of (12) passing through the point $x$ at time $s=t$, that is the solution to

$$
\frac{d}{d s} \gamma(x, t, s)=\lambda(\gamma(x, t, s), s), \quad \gamma(x, t, t)=x .
$$

The function $v(y, s)=u(\gamma(y, 0, s), s)$ is a solution of the initial value problem

$$
\partial_{s} v(y, s)=F\left(v(y, s), \quad v(y, 0)=u_{0}(y),\right.
$$

at least as long as the characteristic curves exist.

Example 8 (The dissipative case) The equation

$$
\begin{aligned}
& \partial_{t} u_{\varepsilon}(x, t)=-u_{\varepsilon}^{3}(x, t), \quad x \in \mathbb{R}, t>0 \\
& u_{\varepsilon}(x, 0)=u_{0 \varepsilon}(x), \quad x \in \mathbb{R}
\end{aligned}
$$

has the solution

$$
u_{\varepsilon}(x, t)=\frac{u_{0 \varepsilon}(x)}{\sqrt{2 t u_{0 \varepsilon}^{2}(x)+1}}=\frac{1}{\sqrt{2 t+1 / u_{0 \varepsilon}^{2}(x)}} .
$$

When the initial data are given by a power of the delta function, $u_{0 \varepsilon}(x)=\varphi_{\varepsilon}^{m}(x)$, the solution formula shows that $u_{\varepsilon}(x, t)$ is a bounded function (uniformly in $\varepsilon$ ) and supported on the line $\{x=0\}$. Thus $u_{\varepsilon}(x, t)$ converges to zero in $\mathcal{D}^{\prime}(\mathbb{R} \times] 0, \infty[)$, and so

$$
\mathcal{S}_{\mathcal{A}}^{\left(a, \mathcal{D}^{\prime}\right)}\left(u_{0}\right)=\left(0,\left[0, m-1[), \quad \mathcal{S}_{\mathcal{A}}^{\left(a, \mathcal{D}^{\prime}\right)}(u)=\varnothing .\right.\right.
$$

Example 9 The equation

$$
\begin{aligned}
& \partial_{t} u_{\varepsilon}(x, t)=\sqrt{1+u_{\varepsilon}^{2}(x, t)}, \quad x \in \mathbb{R}, t>0 \\
& u_{\varepsilon}(x, 0)=u_{0 \varepsilon}(x), \quad x \in \mathbb{R}
\end{aligned}
$$

has the solution

$$
u_{\varepsilon}(x, t)=u_{0 \varepsilon}(x) \cosh t+\sqrt{1+u_{0 \varepsilon}^{2}(x)} \sinh t .
$$


We first take a delta function as initial value, that is, $u_{0 \varepsilon}(x)=\varphi_{\varepsilon}(x)$. Then

$$
\begin{aligned}
\iint u_{\varepsilon}(x, t) \psi(x, t) d x d t & =\iint\left(\varphi(x) \cosh t+\sqrt{\varepsilon^{2}+\varphi^{2}(x)} \sinh t\right) \psi(\varepsilon x, t) d x d t \\
& \rightarrow \iint(\varphi(x) \cosh t+|\varphi(x)| \sinh t) \psi(0, t) d x d t
\end{aligned}
$$

for $\psi \in \mathcal{D}\left(\mathbb{R}^{2}\right)$. Thus in this case

$$
\mathcal{S}_{\mathcal{A}}^{\left(a, \mathcal{D}^{\prime}\right)}\left(u_{0}\right)=\mathcal{S}_{\mathcal{A}}^{\left(a, \mathcal{D}^{\prime}\right)}(u)=\varnothing .
$$

On the other hand, taking the derivative of a delta function as initial value, $u_{0 \varepsilon}(x)=\varphi_{\varepsilon}^{\prime}(x)$, a similar calculation shows that

$$
\iint u_{\varepsilon}(x, t) \psi(x, t) d x d t=\iint\left(\varphi(x) \cosh t+\frac{1}{\varepsilon} \sqrt{\varepsilon^{4}+\left(\varphi^{\prime}\right)^{2}(x)} \sinh t\right) \psi(\varepsilon x, t) d x d t
$$

and so

$$
\mathcal{S}_{\mathcal{A}}^{\left(a, \mathcal{D}^{\prime}\right)}\left(u_{0}\right)=\varnothing, \quad \mathcal{S}_{\mathcal{A}}^{\left(a, \mathcal{D}^{\prime}\right)}(u)=\{(0, t, r): t>0,0 \leq r<1\}
$$

The next example shows that it is quite possible for the singular spectrum to increase with time.

Example 10 The equation

$$
\begin{aligned}
& \left.\partial_{t} u_{\varepsilon}(x, t)=\left(u_{\varepsilon}(x, t)+1\right)\right) \log \left(u_{\varepsilon}(x, t)+1\right), \quad x \in \mathbb{R}, t>0 \\
& u_{\varepsilon}(x, 0)=u_{0 \varepsilon}(x), \quad x \in \mathbb{R}
\end{aligned}
$$

has the solution

$$
u_{\varepsilon}(x, t)=\left(u_{0 \varepsilon}(x)+1\right)^{e^{t}},
$$

provided $u_{0 \varepsilon}>-1$ in which case the function on the right hand side of the differential equation is smooth in the relevant region. To demonstrate the effect, we take a power of the delta function as initial value, that is $u_{0 \varepsilon}(x)=\varphi_{\varepsilon}^{m}(x)$. Then

$$
\mathcal{S}_{\mathcal{A}}^{\left(a, \mathcal{D}^{\prime}\right)}\left(u_{0}\right)=\{(0, r): 0 \leq r<m-1\}, \quad \mathcal{S}_{\mathcal{A}}^{\left(a, \mathcal{D}^{\prime}\right)}(u)=\left\{(0, t, r): t>0,0 \leq r<m e^{t}-1\right\} .
$$

In situations where blow-up in finite time occurs, microlocal asymptotic methods allow to extract information beyond the point of blow-up. This can be done by regularizing the initial data and truncating the nonlinear term. We demonstrate this in a simple situation.

Example 11 Formally, we wish to treat the initial value problem

$$
\begin{aligned}
& \partial_{t} u(x, t)=u^{2}(x, t), \quad x \in \mathbb{R}, t>0 \\
& u(x, 0)=H(x), \quad x \in \mathbb{R}
\end{aligned}
$$

where $H$ denotes the Heaviside function. Clearly, the local solution $u(x, t)=H(x) /(1-t)$ blows up at time $t=1$ when $x>0$. Choose $\chi_{\varepsilon} \in \mathrm{C}^{\infty}(\mathbb{R})$ with

$$
0 \leq \chi_{\varepsilon}(z) \leq 1 ; \chi_{\varepsilon}(z)=1 \text { if }|z| \leq \varepsilon^{-s}, \chi_{\varepsilon}(z)=0 \text { if }|z| \geq 1+\varepsilon^{-s}, s>0 .
$$

Further, let $H_{\varepsilon}(x)=H * \varphi_{\varepsilon}(x)$ where $\varphi_{\varepsilon}$ is a mollifier as in Example 5 . We consider the regularized problem

$$
\begin{aligned}
& \partial_{t} u_{\varepsilon}(x, t)=\chi_{\varepsilon}\left(u_{\varepsilon}(x, t)\right) u_{\varepsilon}^{2}(x, t), \quad x \in \mathbb{R}, t>0 \\
& u_{\varepsilon}(x, 0)=H_{\varepsilon}(x), \quad x \in \mathbb{R} .
\end{aligned}
$$


When $x<0$ and $\varepsilon$ is sufficiently small, $u_{\varepsilon}(x, t)=0$ for all $t \geq 0$. For $x>0, u_{\varepsilon}(x, t)=1 /(1-t)$ as long as $t \leq 1-\varepsilon^{s}$. The cut-off function is chosen in such a way that $\left|\chi_{\varepsilon}(z) z^{2}\right| \leq\left(1+\varepsilon^{-s}\right)^{2}$ for all $z \in \mathbb{R}$. Therefore,

$$
\partial_{t} u_{\varepsilon} \leq\left(1+\varepsilon^{-s}\right)^{2} \text { always and } \partial_{t} u_{\varepsilon}=0 \text { when }\left|u_{\varepsilon}\right| \geq 1+\varepsilon^{-s} .
$$

Continuing the regularized solution beyond time $t=1-\varepsilon^{s}$, we infer by combining the two inequalities that $\varepsilon^{-s} \leq u_{\varepsilon}(x, t) \leq 1+\varepsilon^{-s}$ for $t \geq 1-\varepsilon^{s}$ when $x>0$ and $\varepsilon$ is sufficiently small. Finally, as long as $t<1$, the regularized solution remains bounded with respect to $\varepsilon$ near $(0, t)$ for $\varepsilon$ small enough; after $t=1$, the asymptotic growth of order $\varepsilon^{-s}$ spills over into any neighborhood of every point $(x, t)$ for $x \geq 0$.

Collecting all previous estimates, we obtain the following $\mathrm{C}^{0}$-singular support and $\left(a, \mathrm{C}^{0}\right)$ singular spectrum $\left(\right.$ for $\left.a_{\varepsilon}(r)=\varepsilon^{r}\right)$ of $u=\left[u_{\varepsilon}\right]$ :

$$
\begin{gathered}
\mathcal{S}_{\mathcal{A}}^{\mathrm{C}^{0}}(u)=\mathcal{S}_{1}(u) \cup \mathcal{S}_{2}(u) \text { with } \mathcal{S}_{1}(u)=\{(0, t): 0 \leq t<1\} ; \mathcal{S}_{2}(u)=\{(x, t): x \geq 0, t \geq 1\}, \\
\mathcal{S}_{\mathcal{A}}^{\left(a, \mathrm{C}^{0}\right)}(u)=\left(\mathcal{S}_{1}(u) \times\{0\}\right) \cup\left(\mathcal{S}_{2}(u) \times[0, s]\right) .
\end{gathered}
$$

The $\mathrm{C}^{0}$-singularities (resp. $\left(a, \mathrm{C}^{0}\right)$-singularities) of u are described by means of two sets: $\mathcal{S}_{1}(u)$ and $\mathcal{S}_{2}(u)$ (resp. $\mathcal{S}_{1}(u) \times\{0\}$ and $\left.\mathcal{S}_{2}(u) \times[0, s]\right)$. The set $\mathcal{S}_{1}(u)$ (resp. $\left.\mathcal{S}_{1}(u) \times\{0\}\right)$ is related to the data $\mathrm{C}^{0}$ (resp. $\left(a, \mathrm{C}^{0}\right)$ )-singularity. The set $\mathcal{S}_{2}(u)$ (resp. $\left.\mathcal{S}_{2}(u) \times[0, s]\right)$ is related to the singularity due to the nonlinearity of the equation giving the blow-up at $t=1$. The blow-up locus is the edge $\{x \geq 0, t=1\}$ of $\mathcal{S}_{2}(u)$ and the strength of the blow-up is measured by the length $s$ of the fiber $[0, s]$ above each point of the blow-up locus. This length is closely related to the diameter of the support of the regularizing function $\chi_{\varepsilon}$ and depends essentially on the nature of the blow-up: Changing simultaneously the scales of the regularization and of the cut-off (i.e. replacing $\varepsilon$ by some function $h(\varepsilon) \rightarrow 0$ in the definition of $\varphi_{\varepsilon}$ and $\chi_{\varepsilon}$ ) does not change the fiber and characterizes a sort of moderateness of the strength of the blow-up.

\subsection{The strength of a singularity and the sum law}

When studying the propagation and interaction of singularities in semilinear hyperbolic systems, Rauch and Reed [18] defined the strength of a singularity of a piecewise smooth function. We recall this notion in the one-dimensional case. Assume that the function $f: \mathbb{R} \rightarrow \mathbb{R}$ is smooth on $\left.]-\infty, x_{0}\right]$ and on $\left[x_{0}, \infty\right.$ [ for some point $x_{0} \in \mathbb{R}$. The strength of the singularity of $f$ at $x_{0}$ is the order of the highest derivative which is still continuous across $x_{0}$. For example, if $f$ is continuous with a jump in the first derivative at $x_{0}$, the order is 0 ; if $f$ has a jump at $x_{0}$, the order is -1 . Travers [21] later generalized this notion to include delta functions. Slightly deviating from her definition, but in line with the one of [18], we define the strength of singularity of the $k$-th derivative of a delta function at $x_{0}, \partial_{x}^{k} \delta\left(x-x_{0}\right)$, by $-k-2$.

The significance of these definitions is seen in the description of what Rauch and Reed termed anomalous singularities in semilinear hyperbolic systems. We demonstrate the effect in a paradigmatic example, also due to [18], the $(3 \times 3)$-system

$$
\begin{aligned}
\left(\partial_{t}+\partial_{x}\right) u(x, t) & =0, & & u(x, 0)=u_{0}(x) \\
\left(\partial_{t}-\partial_{x}\right) v(x, t) & =0, & & v(x, 0)=v_{0}(x) \\
\partial_{t} w(x, t) & =u(x, t) v(x, t), & & w(x, 0)=0
\end{aligned}
$$

Assume that $u_{0}$ has a singularity of strength $n_{1} \geq-1$ at $x_{1}=-1$ and $v_{0}$ has a singularity of strength $n_{2} \geq-1$ at $x_{2}=+1$. The characteristic curves emanating from $x_{1}$ and $x_{2}$ are straight lines intersecting at the point $x=0, t=1$. Rauch and Reed showed that, in general, the third component $w$ will have a singularity of strength $n_{3}=n_{1}+n_{2}+2$ along the half-ray $\{(0, t): t \geq 1\}$. This half-ray does not connect backwards to a singularity in the initial data 
for $w$, hence the term anomalous singularity. The formula $n_{3}=n_{1}+n_{2}+2$ is called the sum law. Travers extended this result to the case where $u_{0}$ and $v_{0}$ were given as derivatives of delta functions at $x_{1}$ and $x_{2}$. We are going to further generalize this result to powers of delta functions, after establishing the relation between the strength of a singularity of a function $f$ at $x_{0}$ and the singular spectrum of $f * \varphi_{\varepsilon}$.

We consider a function $f: \mathbb{R} \rightarrow \mathbb{R}$ which is smooth on $\left.]-\infty, x_{0}\right]$ and on $\left[x_{0}, \infty[\right.$ for some point $x_{0} \in \mathbb{R}$; actually only the local behavior near $x_{0}$ is relevant. We fix a mollifier $\varphi_{\varepsilon}(x)=\frac{1}{\varepsilon} \varphi\left(\frac{x}{\varepsilon}\right)$ as in Example 5 and denote the corresponding embedding of $\mathcal{D}^{\prime}(\mathbb{R})$ into the $(\mathcal{C}, \mathcal{E}, \mathcal{P})$-algebra $\mathcal{A}(\mathbb{R})$ by $\iota$. In particular, $\iota(f)=\left[f * \varphi_{\varepsilon}\right]$.

If $f$ is continuous at $x_{0}$, then $\lim _{\varepsilon \rightarrow 0} f * \varphi_{\varepsilon}=f$ in $\mathrm{C}^{0}$. If $f$ has a jump $x_{0}$, this limit does not exist in $\mathrm{C}^{0}$, but $\lim _{\varepsilon \rightarrow 0} \varepsilon^{r} f * \varphi_{\varepsilon}=0$ in $\mathrm{C}^{0}$ for every $r>0$. We have the following result.

Proposition 18 Let $x_{0} \in \mathbb{R}$. If $f: \mathbb{R} \rightarrow \mathbb{R}$ is a smooth function on $\left.]-\infty, x_{0}\right]$ and on $\left[x_{0}, \infty[\right.$ or $f(x)=\partial_{x}^{k} \delta\left(x-x_{0}\right)$ for some $k \in \mathbb{N}$, then the strength of the singularity of $f$ at $x_{0}$ is $-n$ if and only if

$$
\Sigma_{\left(a, \mathrm{C}^{1}\right), x_{0}}(\iota(f))=[0, n] .
$$

Here $n \in \mathbb{N}$ and $a_{\varepsilon}(r)=\varepsilon^{r}$.

Proof. When $n=0$, the function $f$ is continuous and its derivative has a jump at $x_{0}$. From what was said before Proposition 18 it follows that $\Sigma_{\left(a, \mathrm{C}^{1}\right), x_{0}}(\iota(f))=\{0\}$. When $n=1$, the function $f$ has a jump itself at $x_{0}$ and its distributional derivative contains a delta function part. Thus $\lim _{\varepsilon \rightarrow 0} \varepsilon^{r} f * \varphi_{\varepsilon}=0$ in $\mathrm{C}^{0}$ for every $r>0$ and $\lim _{\varepsilon \rightarrow 0} \varepsilon^{r} \partial_{x} f * \varphi_{\varepsilon}=0$ in $\mathrm{C}^{0}$ for every $r>1$, and neither of the two limits exists for smaller $r$. Therefore, $\Sigma_{\left(a, \mathrm{C}^{1}\right), x_{0}}(\iota(f))=[0,1]$. When $n \geq 2, f(x)=\partial_{x}^{n-2} \delta\left(x-x_{0}\right)$ and the assertion is straightforward.

We shall now return to the model equation (13) and demonstrate that the sum law remains valid when the initial data are powers of delta functions. We work in suitable $(\mathcal{C}, \mathcal{E}, \mathcal{P})$-algebras $\mathcal{A}(\mathbb{R})$ and $\mathcal{A}\left(\mathbb{R}^{2}\right)$ in which the initial value problem (13) can be uniquely solved (see the discussion at the beginning of Subsection 4.1). We still consider the scale $a_{\varepsilon}(r)=\varepsilon^{r}$.

Proposition 19 Let $u_{0}(x)=\delta^{m}(x+1), v_{0}(x)=\delta^{n}(x-1)$ for some $m, n \in \mathbb{N}^{*}$. Let $w \in \mathcal{A}\left(\mathbb{R}^{2}\right)$ be the third component of the solution to problem $[13)$. Then $w(x, t)$ vanishes at all points $(x, t)$ with $x \neq 0$ as well as $(0, t)$ with $t<1$, and

$$
\Sigma_{\left(a, \mathrm{C}^{1}\right),(0, t)}(w) \subset[0, m+n]
$$

for $t \geq 1$.

Proof. A representative of $w$ is given by

$$
w_{\varepsilon}(x, t)=\int_{0}^{t} \varphi_{\varepsilon}^{m}(x+1-s) \varphi_{\varepsilon}^{n}(x-1+s) d s .
$$

The fact that the mollifier $\varphi$ has compact support entails that $w_{\varepsilon}(x, t)$ vanishes for sufficiently small $\varepsilon$ whenever $x \neq 0$ or $t<1$. We have

$$
\begin{aligned}
w_{\varepsilon}(x, t) & =\int_{0}^{t} \frac{1}{\varepsilon^{m+n}} \varphi^{m}\left(\frac{x+1-s}{\varepsilon}\right) \varphi^{n}\left(\frac{x-1+s}{\varepsilon}\right) d s \\
\partial_{t} w_{\varepsilon}(x, t) & =\frac{1}{\varepsilon^{m+n}} \varphi^{m}\left(\frac{x+1-t}{\varepsilon}\right) \varphi^{n}\left(\frac{x-1+t}{\varepsilon}\right), \\
\partial_{x} w_{\varepsilon}(x, t) & =\int_{0}^{t} \frac{m}{\varepsilon^{m+n+1}} \varphi^{m-1}\left(\frac{x+1-s}{\varepsilon}\right) \varphi^{\prime}\left(\frac{x+1-s}{\varepsilon}\right) \varphi^{n}\left(\frac{x-1+s}{\varepsilon}\right) d s \\
& +\int_{0}^{t} \frac{n}{\varepsilon^{m+n+1}} \varphi^{m}\left(\frac{x+1-s}{\varepsilon}\right) \varphi^{n-1}\left(\frac{x-1+s}{\varepsilon}\right) \varphi^{\prime}\left(\frac{x-1-s}{\varepsilon}\right) d s .
\end{aligned}
$$


If the support of $\varphi$ is contained in an interval $[-\kappa, \kappa]$, say, then the $t$-integrations extend at most from $x+1-\kappa \varepsilon$ to $x+1+\kappa \varepsilon$ at fixed $x$. Therefore, all terms converge to zero uniformly on $\mathbb{R}^{2}$ when multiplied by $\varepsilon^{r}$ with $r>m+n$. This proves the assertion.

Using the correspondence between the singular spectrum and the strength of a singularity formulated in Proposition 18, as well as Example 5, we may say that the strength of the singularity of $\delta^{m}(x+1)$ at $x_{0}=-1$ is $n_{1}=-m-1$, while the strength of the singularity of $\delta^{n}(x-1)$ at $x_{0}=+1$ is $n_{2}=-n-1$. The strength of the singularity of the solution $w$ at points $(0, t)$ with $t \geq 1$ is $-m-n=n_{1}+n_{2}+2$ and is seen to satisfy the sum law.

\subsection{Regular Colombeau generalized functions}

The subsheaf $\mathcal{G}^{\infty}$ of regular Colombeau functions of the sheaf $\mathcal{G}$ is defined as follows [16]: Given an open subset $\Omega$ of $\mathbb{R}^{d}$, the algebra $\mathcal{G}^{\infty}(\Omega)$ comprises those elements $u$ of $\mathcal{G}(\Omega)$ whose representatives $\left(u_{\varepsilon}\right)_{\varepsilon}$ satisfy the condition

$$
\forall K \Subset \Omega \exists m \in \mathbb{N} \forall l \in \mathbb{N}: p_{K, l}\left(u_{\varepsilon}\right)=o\left(\varepsilon^{-m}\right) \text { as } \varepsilon \rightarrow 0 .
$$

The decisive property is that the bound of order $\varepsilon^{-m}$ is uniform with respect to the order of derivation on compact sets. The algebra $\mathcal{G}^{\infty}(\Omega)$ satisfies $\mathcal{G}^{\infty}(\Omega) \cap \mathcal{D}^{\prime}(\Omega)=\mathrm{C}^{\infty}(\Omega)$ and forms the basis for the investigation of hypoellipticity of linear partial differential operators in the Colombeau framework. We are going to characterize the $\mathcal{G}^{\infty}$-property in terms of the $\mathrm{C}^{\infty}$-singular spectrum. The scale $a$ is still given by $a_{\varepsilon}(r)=\varepsilon^{r}$.

Proposition 20 Let $u \in \mathcal{G}(\Omega)$. Then $u$ belongs to $\mathcal{G}^{\infty}(\Omega)$ if and only if

$$
\Sigma_{\left(a, \mathrm{C}^{\infty}\right), x}(u) \neq \mathbb{R}_{+}
$$

for all $x \in \Omega$.

Proof. If $u \in \mathcal{G}^{\infty}(\Omega), x \in \Omega$ and $V_{x}$ is a relatively compact open neighborhood of $x$, property (14) says that there is $m \in \mathbb{N}$ such that $\lim _{\varepsilon \rightarrow 0} \varepsilon^{m} u_{\varepsilon}=0$ in $\mathrm{C}^{\infty}\left(V_{x}\right)$. Thus $\Sigma_{\left(a, C^{\infty}\right), x}(u) \neq \mathbb{R}_{+}$. Conversely, if $\Sigma_{\left(a, C^{\infty}\right), x}(u) \neq \mathbb{R}_{+}$we can find an open neighborhood $V_{x}$ of $x$ and $m(x) \in \mathbb{N}$ such that $\lim _{\varepsilon \rightarrow 0} \varepsilon^{r} u_{\varepsilon}=0$ in $\mathrm{C}^{\infty}\left(V_{x}\right)$ for all $r \geq m$. Any compact set $K$ can be covered by finitely many such neighborhoods. Letting $m$ be the maximum of the numbers $m(x)$ involved, we obtain property (14).

In relation with regularity theory of solutions to nonlinear partial differential equations, a further subalgebra of $\mathcal{G}(\Omega)$ has been introduced in [17] - the algebra of Colombeau functions of total slow scale type. It consists of those elements $u$ of $\mathcal{G}(\Omega)$ whose representatives $\left(u_{\varepsilon}\right)_{\varepsilon}$ satisfy the condition

$$
\forall K \Subset \Omega \forall r>0 \forall l \in \mathbb{N}: p_{K, l}\left(u_{\varepsilon}\right)=o\left(\varepsilon^{-r}\right) \text { as } \varepsilon \rightarrow 0 .
$$

The term slow scale refers to the fact that the growth is slower than any negative power of $\varepsilon$ as $\varepsilon \rightarrow 0$. This property can again be characterized by means of the singular spectrum.

Proposition 21 An element $u \in \mathcal{G}(\Omega)$ is of total slow scale type if and only if

$$
\Sigma_{\left(a, \mathrm{C}^{\infty}\right), x}(u) \subset\{0\}
$$

for all $x \in \Omega$.

Proof. If $u$ is of total slow scale type, $x \in \Omega$ and $V_{x}$ is a relatively compact open neighborhood of $x$, property (15) implies that $\lim _{\varepsilon \rightarrow 0} \varepsilon^{s} u_{\varepsilon}=0$ in $\mathrm{C}^{\infty}\left(V_{x}\right)$ for every $s>0$. Thus $\Sigma_{\left(a, \mathrm{C}^{\infty}\right), x}(u) \subset\{0\}$. To prove the converse, we take a compact subset $K$ and $r>0$ and cover $K$ by finitely many neighborhoods $V_{x}$ of points $x \in K$ such that $\lim _{\varepsilon \rightarrow 0} \varepsilon^{r} u_{\varepsilon}=0$ in $\mathrm{C}^{\infty}\left(V_{x}\right)$. Then property (15) follows. 


\section{References}

[1] Aragona, J., Biagioni, H.: Intrinsic definition of the Colombeau algebra of generalized functions. Anal. Math. 17, 75-132 (1991)

[2] Colombeau, J.F.: Multiplication of Distributions: a tool in mathematics, numerical engineering and theoretical physics. Lecture Notes in Mathematics, vol. 1532. Springer-Verlag, Berlin (1992)

[3] Delcroix, A: Remarks on the embedding of spaces of distributions into spaces of Colombeau generalized functions. Novi Sad J. Math. 35(2), 27-40 (2005)

[4] Delcroix, A.: Regular rapidly decreasing nonlinear generalized functions. Application to microlocal regularity. J. Math. Anal. Appl. 327, 564-584 (2007)

[5] Garetto, C., Gramchev, T., Oberguggenberger, M.: Pseudo-Differential operators and regularity theory. Electron J. Diff. Eqns. 2005(116), 1-43 (2005)

[6] Garetto, C., Hörmann, G.: Microlocal analysis of generalized functions: pseudodifferential techniques and propagation of singularities. Proc. Edinb. Math. Soc. 48, 603-629 (2005)

[7] Grosser, M., Kunzinger, M., Oberguggenberger, M., Steinbauer, R.: Geometric Theory of Generalized Functions with Applications to General Relativity. Kluwer Academic Publ., Dordrecht (2001)

[8] Hörmander, L.: The Analysis of Linear Partial Differential Operators, I: Distribution Theory and Fourier Analysis. Grundlehren der mathematischen Wissenschaften, vol. 256, 2nd edition. Springer-Verlag, Berlin (1990)

[9] Hörmann, G., De Hoop, M.V.: Microlocal analysis and global solutions for some hyperbolic equations with discontinuous coefficients. Acta Appl. Math. 67, 173-224 (2001)

[10] Hörmann, G., Kunzinger, M.: Microlocal properties of basic operations in Colombeau algebras. J. Math. Anal. Appl. 261, 254-270 (2001)

[11] Hörmann, G., Oberguggenberger, M., Pilipović, S.: Microlocal hypoellipticity of linear differential operators with generalized functions as coefficients. Trans. Amer. Math. Soc. 358, 3363-3383 (2006)

[12] Marti, J.A.: $(\mathcal{C}, \mathcal{E}, \mathcal{P})$-Sheaf structure and applications. In: Grosser, M., Hörmann, G., Kunzinger, M., Oberguggenberger, M. (Eds.) Nonlinear Theory of Generalized Functions. Chapman \& Hall/CRC Research Notes in Mathematics, vol. 401, pp.175-186. Boca Raton (1999)

[13] Marti, J.A.: Nonlinear algebraic analysis of delta shock wave to Burgers' equation. Pacific J. Math. 210(1), 165-187 (2003)

[14] Marti, J.A.: $\mathcal{G}^{L}$-microanalysis of generalized functions. Integral Transf. Spec. Funct. 2-3, $119-125$ (2006)

[15] Nedeljkov, M., Pilipović, S., Scarpalézos, D.: The linear theory of Colombeau generalized functions. Pitman Research Notes in Mathematics, vol. 385. Longman Scientific \& Technical, Harlow (1998)

[16] Oberguggenberger, M.: Multiplication of Distributions and Applications to Partial Differential Equations. Pitman Research Notes in Mathematics, vol. 259. Longman Scientific \& Technical, Harlow (1992) 
[17] Oberguggenberger, M.: Generalized solutions to nonlinear wave equations. Matemática Contemporânea 27, 169-187 (2004)

[18] Rauch, J., Reed, M.: Jump discontinuities of semilinear, strictly hyperbolic equations in two variables: Creation and propagation. Comm. Math. Phys. 81, 203-227 (1981)

[19] Scarpalézos, D.: Colombeau's generalized functions: topological structures; microlocal properties. A simplified point of view. Bull. Cl. Sci. Math. Nat. Sci. Math. 25, 89-114 (2000)

[20] Schwartz, L.: Théorie des Distributions. Hermann, Paris (1966)

[21] Travers, K.: Semilinear hyperbolic systems in one space dimension with strongly singular initial data. Electron. J. Diff. Eqns. 1997(14), 1-11 (1997) 